\title{
THIN LAYER MODELS FOR ELECTROMAGNETISM
}

\author{
MARC DURUFLÉ, VICTOR PÉRON, AND CLAIR POIGNARD
}

\begin{abstract}
We present a review on the accuracy of asymptotic models for the scattering problem of electromagnetic waves in domains with thin layer. These models appear as first order approximations of the electromagnetic field. They are obtained thanks to a multiscale expansion of the exact solution with respect to the thickness of the thin layer, that makes possible to replace the thin layer by approximate conditions. We present the advantages and the drawbacks of several approximations together with numerical validations and simulations. The main motivation of this work concerns the computation of electromagnetic field in biological cells. The main difficulty to compute the local electric field lies in the thinness of the membrane and in the high contrast between the electrical conductivities of the cytoplasm and of the membrane, which provides a specific behavior of the electromagnetic field at low frequencies.
\end{abstract}

\section{CONTENTS}

1. Introduction 1

2. The Mathematical Model 3

3. Multiscale Expansion $\quad 5$

4. Generalized Impedance Transmission Conditions 9

5. Numerical Simulations 13

$\begin{array}{ll}\text { 6. Conclusion } & 19\end{array}$

References $\quad 22$

\section{INTRODUCTION}

The aim of this work is to provide a review of several asymptotic models for the scattering problem of time-harmonic electromagnetic waves in domains with thin layer. Media with thin inclusions appear in many domains: geophysical applications, microwave imaging, biomedical applications, cell phone radiations, radar applications, non-destructive testing... In this paper, the simplified configuration is mainly motivated by the computation of the electromagnetic field in biological cells.

The electromagnetic modeling of biological cells has become extremely important since several years, in particular in the biomedical research area. In the simple model of Fear, and Stuchly or Foster and Schwan $[8,9,10]$, the biological cell is composed of a conducting cytoplasm surrounded by a thin insulating membrane. When the cell is exposed to an electric field, the local field near the membrane may overcome physiological values. Then, complex phenomenon known as electropermeabilization (or electroporation) may occur [21]: the cell membrane

1991 Mathematics Subject Classification. 34E05, 34E10, $58 \mathrm{~J} 37$.

Key words and phrases. asymptotics, time-harmonic Maxwell's equations, finite element method, edge elements. 
is destructured and some outer molecules might be internalized inside the cell, as described in the model of Kavian et al. [11]. These process hold great promises in oncology and gene therapy, particularly, to deliver drug molecules in cancer treatment. This is the reason why several papers in the bioelectromagnetic research area deal with numerical modeling of the cell (see for instance $[12,20,19])$ and with numerical computations of the membrane voltage. Actually, the main difficulties of the calculation of the local electric field lie in the thinness of the membrane and in the high contrast between the electromagnetic properties of the cytoplasm and the membrane. More precisely, though the electric permittivities of these two media are of the same order of magnitude, the membrane conductivity is much lower than the cytoplasm conductivity, which provides particular behavior of the electromagnetic field at low frequencies, for which the condution currents predominate.

In previous papers $[18,16,17,15]$, Poignard et al. have proposed an asymptotic analysis to compute the solution to the conductivity problem, the so-called electric potential, in domains with thin layer. In particular, Perrussel and Poignard have derived the asymptotic expansion of the electric potential at any order in domains with resistive thin layer [14]. More recently, we have derived an asymptotic model for the solution to time-harmonic Maxwell equations, the so-called electromagnetic field, in biological cell at mid-frequency [7, Eq. (5.1)]. In the proceeding [6, Sec. 3], we have derived an asymptotic model for the electromagnetic field in biological cell at low-frequency which corresponds to a resistive membrane. All these papers are based on a multiscale asymptotic expansion of the partial differential equations, that makes possible to replace the thin layer by appropriate transmission conditions.

In [7], we have derived a multi-scale expansion for the electric field in power series of a small parameter $\varepsilon$, which represents the relative size of the cell membrane [7, Eq. (5.1)]. We inferred appropriate transmission conditions "at first order" on the boundary of the cytoplasm satisfied by the first two terms of the expansion $\mathbf{E}^{0}+\varepsilon \mathbf{E}^{1}$. We proved uniform estimates (in energy norm) with respect to $\varepsilon$ for the error between the exact solution $\mathbf{E}^{\varepsilon}$ and the approximate solution $\mathbf{E}^{0}+\varepsilon \mathbf{E}^{1}[7$, Th. 2.9]. We validated the asymptotic expansion up to the first two terms, proving estimates for the remainder of the expansion defined as $\mathbf{E}^{\varepsilon}-\left(\mathbf{E}^{0}+\varepsilon \mathbf{E}^{1}\right)$ [7, Th. 6.3]. We recall in Sec. 3.1 the two first order of this asymptotic expansion which is relevant in the mid-frequency range. This expansion is no longer valid in the low-frequency regime. We recall in Sec. 3.2 the asymptotic expansion and the resistive model [6] adapted to this frequency range.

Recently, Delourme et al. have derived an asymptotic model [4, Eqs. (1)-(5)] for the electrical field in a domain with a periodic oscillating thin (and straight) layer. The authors proved that this asymptotic model is a second order model [4, Prop. 19]. The authors proved this result by exhibiting a Helmholtz decomposition adapted to the variational space of this model [4, Prop. 9]. This Helmholtz decomposition holds under a spectral assumption [4, Hyp. 6].

The aim of this paper is to present a review on the accuracy of different approximations of the electromagnetic field in time-harmonic regime with thin layer. We present the advantages and the drawbacks of these approximations and we make the link with the previous work of [4] and [2]. This paper is focused on the numerical aspects. The main tools to derive theoretical accuracy of the approximations have already been presented in [7, 4].

The GITC models are new models. We present in Sec. 4 the GITC model of order 2 (11). We have made explicit the model of Delourme et al. [4, Eqs. (1)-(5)] in the case of an homogeneous thin layer. It corresponds to the GITC model (11) for a specific choice of parameters $\alpha=\beta=\frac{1}{2}$ introduced in Sec. 3.3. We introduce in Sec. 4.1 a new unified formulation, which involves 
the coefficients $A,_{\beta}, B_{\beta}, C_{\beta}$, and $D_{\beta}$ in (9), in order to write the different models in the same framework. This simplifies the comparison between the models.

In Sec. 5, we show the numerical accuracy of the different asymptotic models for the diffraction problem of a penetrable sphere surrounded by a conductind thin layer. Then, we apply the different models to the case of a spherical biological cell: the membrane is then very resistive. We observe a mid-frequency regime (for which GITCs provide a second order approximation) and a low-frequency regime (for which only a first order approximation is observed for GITCs). We discuss advantages and drawbacks for all these models in the conclusion.

\section{The Mathematical Model}

In this section, we introduce the mathematical framework and the exact model that will be approximated in the next sections.

2.1. Notations. For any orientable smooth surface without boundary $\mathcal{S}$ of $\mathbb{R}^{3}$, the unit normal vector $\mathbf{n}$ on $\mathcal{S}$ is outwardly oriented from the interior domain enclosed by $\mathcal{S}$ towards the outer domain.

We denote by $\operatorname{curl}_{\mathcal{S}}$ the tangential rotational operator (which applies to functions defined on $\mathcal{S}$ ) and $\operatorname{curl}_{\mathcal{S}}$ the surface rotational operator (which applies to vector fields) [13] :

$$
\begin{gathered}
\forall f \in C^{\infty}(\mathcal{S}), \quad \operatorname{curl}_{\mathcal{S}} f=\left(\nabla_{\mathcal{S}} f\right) \times \mathbf{n}, \\
\forall \mathbf{v} \in\left(C^{\infty}(\mathcal{S})\right)^{3}, \quad \operatorname{curl}_{\mathcal{S}} \mathbf{v}=\operatorname{div}_{\mathcal{S}}(\mathbf{v} \times \mathbf{n}),
\end{gathered}
$$

where $\nabla_{\mathcal{S}}$ and $\operatorname{div}_{\mathcal{S}}$ are respectively the tangential gradient and the surface divergence on $\mathcal{S}$. We denote respectively by $\mathrm{TH}^{-1 / 2}\left(\operatorname{div}_{\mathcal{S}}, \mathcal{S}\right)$ and $\mathrm{TH}\left(\operatorname{curl}_{\mathcal{S}}, \mathcal{S}\right)$ the spaces of tangent vector fields of the above operators $\operatorname{div}_{\mathcal{S}}$ and $\operatorname{curl}_{\mathcal{S}}[13]$ :

$$
\begin{aligned}
& \mathrm{TH}^{-1 / 2}\left(\operatorname{div}_{\mathcal{S}}, \mathcal{S}\right)=\left\{\mathbf{v} \in \mathbf{H}^{-1 / 2}(\mathcal{S}), \operatorname{div}_{\mathcal{S}} \mathbf{v} \in \mathrm{H}^{-1 / 2}(\mathcal{S})\right\}, \\
& \mathrm{TH}\left(\operatorname{curl}_{\mathcal{S}}, \mathcal{S}\right)=\left\{\mathbf{v} \in \mathbf{L}^{2}(\mathcal{S}), \operatorname{curl}_{\mathcal{S}} \mathbf{v} \in \mathrm{L}^{2}(\mathcal{S})\right\},
\end{aligned}
$$

where $\mathbf{L}^{2}(\mathcal{S})=\mathrm{L}^{2}(\mathcal{S})^{3}$ and $\mathbf{H}^{-1 / 2}(\mathcal{S})=\mathrm{H}^{-1 / 2}(\mathcal{S})^{3}$.

Equipped with their graph norm, $\mathrm{TH}^{-1 / 2}\left(\operatorname{div}_{\mathcal{S}}, \mathcal{S}\right)$ and $\mathrm{TH}\left(\operatorname{curl}_{\mathcal{S}}, \mathcal{S}\right)$ are Hilbert spaces. We denote by $\left.\mathbf{v}_{\mathrm{T}}\right|_{\mathcal{S}}$ the tangent component of the vector field $\mathbf{v}$ defined in a neighborhood of $\mathcal{S}$ :

$$
\left.\mathbf{v}_{\mathrm{T}}\right|_{\mathcal{S}}=\mathbf{n} \times\left(\left.\mathbf{v}\right|_{\mathcal{S}} \times \mathbf{n}\right),
$$

and we denote by $[\mathbf{v}]_{\mathcal{S}}$ the jump of $\mathbf{v}$ across $\mathcal{S}$ :

$$
[\mathbf{v}]_{\mathcal{S}}=\left.\mathbf{v}\right|_{\mathcal{S}^{+}}-\left.\mathbf{v}\right|_{\mathcal{S}^{-}} .
$$

2.2. Time-harmonic Maxwell equation in single cell. Biological cells consist of a cytoplasm surrounded by a thin resistive layer. Throughout the paper we denote by $\mathcal{O}$ the domain of interest which is composed of the outer cell medium and the cell. Let us denote by $\mathcal{O}_{c}$ the cell cytoplasm, and by $\mathcal{O}_{\mathrm{m}}^{\varepsilon}$ the cell membrane surrounding $\mathcal{O}_{\mathrm{c}}$, whose thickness is constant and denoted by $\varepsilon$. Assuming, without loss of generality, that the domain $\mathcal{O}_{\mathrm{c}}$ is independent of $\varepsilon$, the extracellular domain is then $\varepsilon$-dependent. We denote it by $\mathcal{O}_{\mathrm{e}}^{\varepsilon}$, in a such way that (see Figure 1) :

$$
\mathcal{O}=\mathcal{O}_{\mathrm{c}} \cup \overline{\mathcal{O}_{\mathrm{m}}^{\varepsilon}} \cup \mathcal{O}_{\mathrm{e}}^{\varepsilon} \text {. }
$$

The boundary of the cytoplasm is the smooth surface denoted by $\Gamma$ while $\Gamma^{\varepsilon}$ is the cell boundary, i.e. $\Gamma^{\varepsilon}$ is the boundary of $\overline{\mathcal{O}_{\mathrm{c}}} \cup \mathcal{O}_{\mathrm{m}}^{\varepsilon}$. 


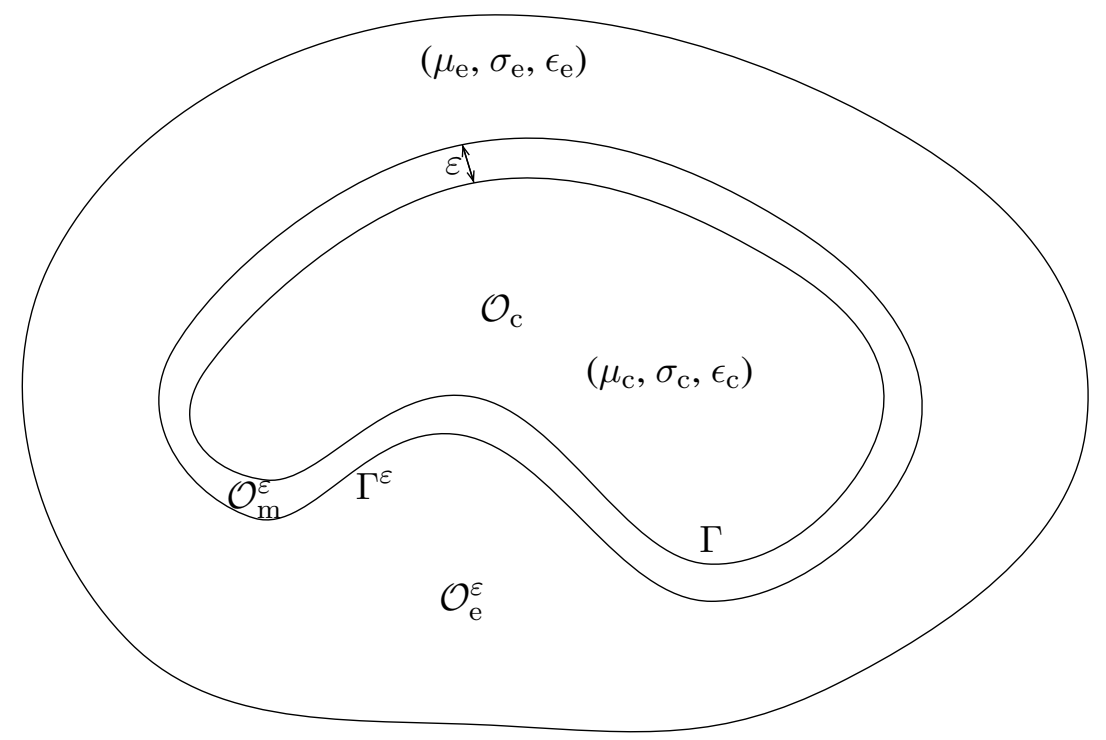

FIGURE 1. A cross-section of the domain $\mathcal{O}$ and its subdomains $\mathcal{O}_{\mathrm{c}}, \mathcal{O}_{\mathrm{m}}^{\varepsilon}, \mathcal{O}_{\mathrm{e}}$

The electromagnetic properties of $\mathcal{O}$ are given by the following piecewise-constant functions $\mu, \underline{\epsilon}$, and $\underline{\sigma}$ corresponding to the respective magnetic permeability, electrical permittivity, and conductivity of $\mathcal{O}$ :

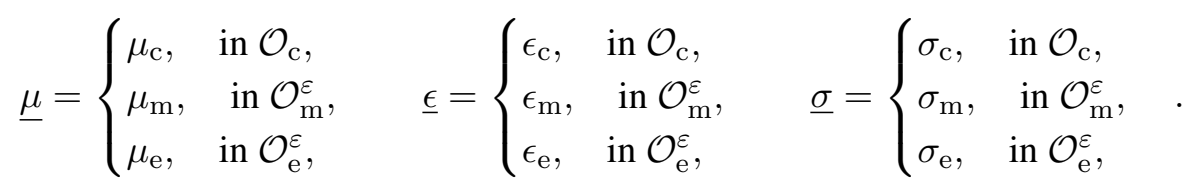

Let us denote by $\mathbf{J}$ the time-harmonic current source and let $\omega$ be the frequency. For the sake of simplicity, we assume that $\mathbf{J}$ is smooth , supported in $\mathcal{O}_{\mathrm{e}}^{\varepsilon}$ and that it vanishes in a neighborhood of the cell membrane. Maxwell's equations link the electric field $\mathbf{E}$ and the magnetic field $\mathbf{H}$, through Faraday's and Ampère's laws in $\mathcal{O}$ :

$$
\operatorname{curl} \mathbf{E}^{\varepsilon}-i \omega \underline{\mu} \mathbf{H}^{\varepsilon}=0 \text { and } \operatorname{curl} \mathbf{H}^{\varepsilon}+(i \omega \underline{\epsilon}-\underline{\sigma}) \mathbf{E}^{\varepsilon}=\mathbf{J} \quad \text { in } \mathcal{O} .
$$

We complement this problem with a Silver-Müller boundary condition set on $\partial \mathcal{O}$. Denoting by $\underline{\kappa}$ the complex wave number given by

$$
\forall x \in \mathcal{O}, \quad \underline{\kappa}^{2}(x)=\omega^{2} \underline{\mu}(x)\left(\underline{\epsilon}(x)+i \frac{\underline{\sigma}(x)}{\omega}\right), \quad \Im(\underline{\kappa}(x)) \geq 0,
$$

Maxwell's system of first order partial differential equations can be reduced to the following second-order equation

$$
\operatorname{curlcurl} \mathbf{E}^{\varepsilon}-\underline{\kappa}^{2} \mathbf{E}^{\varepsilon}=i \omega \underline{\mu} \mathbf{J} \quad \text { in } \quad \mathcal{O}_{\mathrm{c}} \cup \mathcal{O}_{\mathrm{m}}^{\varepsilon} \cup \mathcal{O}_{\mathrm{e}}^{\varepsilon},
$$


with the following transmission conditions across $\Gamma$ and $\Gamma^{\varepsilon}$

$$
\begin{aligned}
& \mathbf{E}_{\mathrm{e}}^{\varepsilon} \times\left.\mathbf{n}\right|_{\Gamma^{\varepsilon}}=\mathbf{E}_{\mathrm{m}}^{\varepsilon} \times\left.\mathbf{n}\right|_{\Gamma^{\varepsilon}}, \quad \frac{1}{\mu_{\mathrm{e}}} \operatorname{curl} \mathbf{E}_{\mathrm{e}}^{\varepsilon} \times\left.\mathbf{n}\right|_{\Gamma^{\varepsilon}}=\frac{1}{\mu_{\mathrm{m}}} \operatorname{curl} \mathbf{E}_{\mathrm{m}}^{\varepsilon} \times\left.\mathbf{n}\right|_{\Gamma^{\varepsilon}}, \\
& \mathbf{E}_{\mathrm{c}}^{\varepsilon} \times\left.\mathbf{n}\right|_{\Gamma}=\mathbf{E}_{\mathrm{m}}^{\varepsilon} \times\left.\mathbf{n}\right|_{\Gamma}, \quad \frac{1}{\mu_{\mathrm{c}}} \operatorname{curl} \mathbf{E}_{\mathrm{c}}^{\varepsilon} \times\left.\mathbf{n}\right|_{\Gamma}=\frac{1}{\mu_{\mathrm{m}}} \operatorname{curl} \mathbf{E}_{\mathrm{m}}^{\varepsilon} \times\left.\mathbf{n}\right|_{\Gamma},
\end{aligned}
$$

where $\mathbf{E}_{\mathrm{e}}^{\varepsilon}, \mathbf{E}_{\mathrm{m}}^{\varepsilon}, \mathbf{E}_{\mathrm{c}}^{\varepsilon}$ denote the respective restrictions of $\mathbf{E}^{\varepsilon}$ to the domains $\mathcal{O}_{\mathrm{e}}^{\varepsilon}, \mathcal{O}_{\mathrm{m}}^{\varepsilon}$ and $\mathcal{O}_{\mathrm{c}}$. The boundary condition is given as

$$
\operatorname{curl} \mathbf{E}^{\varepsilon} \times \mathbf{n}-i \kappa_{\mathrm{e}} \mathbf{n} \times \mathbf{E}^{\varepsilon} \times \mathbf{n}=0 \quad \text { on } \quad \partial \mathcal{O} .
$$

\section{Multiscale ExPAnsion}

In order to avoid the meshing of the thin membrane, it is convenient to approximate the solution to problem (1), by replacing the thin layer by appropriate conditions across the surface $\Gamma$. The idea, as presented in [7], consists in rewriting the operator curl curl in the domain $\mathcal{O}_{\mathrm{m}}^{\varepsilon}$ in local coordinates $\left(\mathbf{x}_{\mathbf{T}}, x_{3}\right)[1,7]$. The variable $x_{3} \in(0, \varepsilon)$ is the Euclidean distance to $\Gamma$ and $\mathbf{x}_{\mathbf{T}}$ denotes tangential coordinates on $\Gamma$. Then performing the change of variable $x_{3}=\varepsilon \eta$, rewriting the operator in $\left(\mathbf{x}_{\mathbf{T}}, \eta\right)$-coordinates and assuming that $\mathbf{E}^{\varepsilon}$ can be developed as a formal expansion in power series of $\varepsilon$, we obtain the approximation of $\mathbf{E}^{\varepsilon}$ at the desired order of accuracy. The rigorous derivation of the expansion is not in the scope of the present paper and we refer the reader to [7] for a detailed description of the calculation. Only the two first terms of the multiscale expansion are presented in this paper, namely:

$$
\begin{aligned}
& \mathbf{E}^{\varepsilon} \approx \mathbf{E}^{0}+\varepsilon \mathbf{E}^{1}, \quad \text { in } \quad \mathcal{O}_{\mathrm{c}} \cup \mathcal{O}_{\mathrm{e}} \\
& \mathbf{E}^{\varepsilon} \approx \mathbf{E}_{\mathrm{m}}^{0}\left(\mathbf{x}_{\mathbf{T}}, \frac{x_{3}}{\varepsilon}\right)+\varepsilon \mathbf{E}_{\mathrm{m}}^{1}\left(\mathbf{x}_{\mathbf{T}}, \frac{x_{3}}{\varepsilon}\right), \quad \text { for almost any } \quad\left(\mathbf{x}_{\mathbf{T}}, x_{3}\right) \in \Gamma \times(0, \varepsilon),
\end{aligned}
$$

where $\mathcal{O}_{\mathrm{e}}$ denotes the domain $\mathcal{O}_{\mathrm{e}}=\mathcal{O} \backslash \overline{\mathcal{O}_{\mathrm{c}}}$. For such a purpose it is convenient to introduce the electromagnetic properties of the "background" problem, i.e the domain $\mathcal{O}$ without the membrane:

$$
\mu=\left\{\begin{array}{ll}
\mu_{\mathrm{c}}, & \text { in } \mathcal{O}_{\mathrm{c}}, \\
\mu_{\mathrm{e}}, & \text { in } \mathcal{O}_{\mathrm{e}},
\end{array} \quad \epsilon=\left\{\begin{array}{ll}
\epsilon_{\mathrm{c}}, & \text { in } \mathcal{O}_{\mathrm{c}}, \\
\epsilon_{\mathrm{e}}, & \text { in } \mathcal{O}_{\mathrm{e}},
\end{array} \quad \sigma=\left\{\begin{array}{ll}
\sigma_{\mathrm{c}}, & \text { in } \mathcal{O}_{\mathrm{c}}, \\
\sigma_{\mathrm{e}}, & \text { in } \mathcal{O}_{\mathrm{e}},
\end{array},\right.\right.\right.
$$

and we define similarly $\kappa$ as

$$
\kappa=\left\{\begin{array}{ll}
\kappa_{\mathrm{c}}, & \text { in } \mathcal{O}_{\mathrm{c}}, \\
\kappa_{\mathrm{e}}, & \text { in } \mathcal{O}_{\mathrm{e}},
\end{array} .\right.
$$

It is worth noting that even in the linear regime, biological cell is a complex material, which behaves differently when the frequency of the excitation changes.

Actually, if the complex constants $\kappa_{\mathrm{e}}$ and $\kappa_{\mathrm{c}}$ are of similar order, for some frequencies we call low-frequency range, the modulus $\left|\kappa_{\mathrm{m}} / \kappa_{\mathrm{e}}\right|^{2}$ is small and of order similar to $\varepsilon$, while for higher frequencies, called mid-frequency range, $\kappa_{\mathrm{m}}, \kappa_{\mathrm{e}}$ (and thus $\kappa_{\mathrm{c}}$ ) are of the same order. We refer to [16] for more details. Therefore the asymptotic expansion has to take this feature into account.

3.1. The mid-frequency case. In the mid-frequency range, the cell is a soft contrast material. In this section, we recall the first terms of the expansion given in [7]. 
Two first orders of the asymptotic expansion. The first term $\mathbf{E}^{0}$ of the expansion satisfies the problem without the layer:

$$
\begin{aligned}
& \text { curl curl } \mathbf{E}^{0}-\kappa_{\mathrm{e}}^{2} \mathbf{E}^{0}=i \omega \mu_{\mathrm{e}} \mathbf{J}, \text { in } \mathcal{O}_{\mathrm{e}}, \\
& \text { curl curl } \mathbf{E}^{0}-\kappa_{\mathrm{c}}^{2} \mathbf{E}^{0}=0, \text { in } \mathcal{O}_{\mathrm{c}},
\end{aligned}
$$

with the transmission conditions:

$$
\left[\mathbf{E}^{0} \times \mathbf{n}\right]_{\Gamma}=0, \quad \frac{1}{\mu_{\mathrm{e}}} \operatorname{curl} \mathbf{E}^{0} \times\left.\mathbf{n}\right|_{\Gamma^{+}}=\frac{1}{\mu_{\mathrm{c}}} \operatorname{curl} \mathbf{E}^{0} \times\left.\mathbf{n}\right|_{\Gamma^{-}},
$$

and the Silver-Müller condition

$$
\operatorname{curl} \mathbf{E}^{0} \times \mathbf{n}-i \kappa_{\mathrm{e}} \mathbf{n} \times \mathbf{E}^{0} \times \mathbf{n}=0 \quad \text { on } \quad \partial \mathcal{O} .
$$

The influence of the thin layer appears in the problem satisfied by the second term $\mathbf{E}^{1}$. According to [7], it is necessary to introduce the following tangent operators $\mathbf{T}$ and $\mathbf{S}$ on $\Gamma^{1}$ :

(3) $\mathbf{T}(\mathbf{E})=\left(\frac{\mu_{\mathrm{m}}}{\kappa_{\mathrm{m}}^{2}}-\frac{\mu_{\mathrm{e}}}{\kappa_{\mathrm{e}}^{2}}\right) \mathbf{n} \times\left.\operatorname{curl}_{\Gamma} \operatorname{curl}_{\Gamma}\left(\frac{1}{\mu_{\mathrm{c}}} \operatorname{curl} \mathbf{E}\right)_{\mathrm{T}}\right|_{\Gamma^{-}}+\left.\left(\mu_{\mathrm{m}}-\mu_{\mathrm{e}}\right) \frac{1}{\mu_{\mathrm{c}}}(\operatorname{curl} \mathbf{E} \times \mathbf{n})\right|_{\Gamma^{-}}$,

(4) $\mathbf{S}(\mathbf{E})=-\left.\left(\frac{\kappa_{\mathrm{m}}^{2}}{\mu_{\mathrm{m}}}-\frac{\kappa_{\mathrm{e}}^{2}}{\mu_{\mathrm{e}}}\right)(\mathbf{n} \times \mathbf{E} \times \mathbf{n})\right|_{\Gamma^{+}}+\left.\left(\frac{1}{\mu_{\mathrm{m}}}-\frac{1}{\mu_{\mathrm{e}}}\right) \overrightarrow{c u r l}_{\Gamma} \operatorname{curl}_{\Gamma}(\mathbf{n} \times \mathbf{E} \times \mathbf{n})\right|_{\Gamma^{+}}$.

such as $\mathbf{E}^{1}$ satisfies

$$
\begin{aligned}
& \text { curl curl } \mathbf{E}^{1}-\kappa^{2} \mathbf{E}^{1}=0, \text { in } \mathcal{O}_{\mathrm{c}} \cup \mathcal{O}_{\mathrm{e}}, \\
& \operatorname{curl} \mathbf{E}^{1} \times \mathbf{n}-i \kappa_{\mathrm{e}} \mathbf{n} \times \mathbf{E}^{1} \times \mathbf{n}=0 \quad \text { on } \quad \partial \mathcal{O},
\end{aligned}
$$

with the following transmission conditions on $\Gamma$

$$
\begin{aligned}
& \mathbf{n} \times\left.\mathbf{E}^{1}\right|_{\Gamma^{+}} \times \mathbf{n}=\mathbf{n} \times\left.\mathbf{E}^{1}\right|_{\Gamma^{-}} \times \mathbf{n}+\mathbf{T}\left(\mathbf{E}^{0}\right), \\
& \left.\frac{1}{\mu_{\mathrm{e}}}\left(\operatorname{curl} \mathbf{E}^{1} \times \mathbf{n}\right)\right|_{\Gamma^{+}}=\left.\frac{1}{\mu_{\mathrm{c}}}\left(\operatorname{curl} \mathbf{E}^{1} \times \mathbf{n}\right)\right|_{\Gamma^{-}}+\mathbf{S}\left(\mathbf{E}^{0}\right) .
\end{aligned}
$$

3.2. The low-frequency case. For low frequencies, the ratio $\left|\kappa_{\mathrm{m}} / \kappa_{\mathrm{e}}\right|^{2}$ is of order $\varepsilon$. It is convenient to introduce the complex $\widetilde{\kappa}_{\mathrm{m}}$ such as

$$
\kappa_{\mathrm{m}}^{2}=\varepsilon \widetilde{\kappa}_{\mathrm{m}}^{2},
$$

where $\widetilde{\kappa}_{\mathrm{m}}$ is such that its imaginary and real parts have the same sign as these of $\kappa_{\mathrm{m}}$.

We perform an asymptotic expansion as done in [7] by assuming that $\widetilde{\kappa}_{\mathrm{m}}$ is independent of $\varepsilon$, instead of assuming that $\kappa_{\mathrm{m}}$ is independent of $\varepsilon$ in [7]. Thus the derivation of the expansion becomes [6]:

$$
\begin{aligned}
& \mathbf{E}^{\varepsilon} \approx \mathbf{E}^{0}, \quad \text { in } \quad \mathcal{O}_{\mathrm{c}} \cup \mathcal{O}_{\mathrm{e}} \\
& \mathbf{E}^{\varepsilon} \approx \varepsilon^{-1} \mathbf{E}_{\mathrm{m}}^{-1}\left(\mathbf{x}_{\mathrm{T}}, \frac{x_{3}}{\varepsilon}\right)+\mathbf{E}_{\mathrm{m}}^{0}\left(\mathbf{x}_{\mathbf{T}}, \frac{x_{3}}{\varepsilon}\right), \quad \text { for almost any } \quad\left(\mathbf{x}_{\mathrm{T}}, x_{3}\right) \in \Gamma \times(0, \varepsilon) .
\end{aligned}
$$

Unlike the mid-frequency case, this expansion starts at the order $\varepsilon^{-1}$ in the thin layer.

\footnotetext{
${ }^{1}$ In the reference [7, Eq. (18)], there is a sign error in front of the term $\left.\left(\frac{\kappa_{\mathrm{m}}^{2}}{\mu_{\mathrm{m}}}-\frac{\kappa_{\mathrm{e}}^{2}}{\mu_{\mathrm{e}}}\right)(\mathbf{n} \times \mathbf{E} \times \mathbf{n})\right|_{\Gamma^{+}}$which appears in the expression of $\mathbf{S}(\mathbf{E})$.
} 
This process has been presented in [6]. It has been found that at low frequency, the membrane influence appears at the zeroth order term, meaning that the membrane influence should not be neglected, namely the limit field $\mathbf{E}^{0}$ satisfies the problem

$$
\begin{aligned}
& \text { curlcurl } \mathbf{E}^{0}-\kappa_{\mathrm{e}}^{2} \mathbf{E}^{0}=i \omega \mu_{\mathrm{e}} \mathbf{J}, \quad \text { in } \mathcal{O}_{\mathrm{e}}, \\
& \text { curl curl } \mathbf{E}^{0}-\kappa_{\mathrm{c}}^{2} \mathbf{E}^{0}=0, \quad \text { in } \mathcal{O}_{\mathrm{c}}, \\
& \operatorname{curl} \mathbf{E}^{0} \times \mathbf{n}-i \kappa_{\mathrm{e}} \mathbf{n} \times \mathbf{E}^{0} \times \mathbf{n}=0, \quad \text { on } \partial \mathcal{O},
\end{aligned}
$$

with the following transmission conditions set on $\Gamma$

$$
\begin{gathered}
\left.\frac{1}{\mu_{\mathrm{e}}} \operatorname{curl} \mathbf{E}^{0}\right|_{\Gamma^{+}} \times \mathbf{n}=\left.\frac{1}{\mu_{\mathrm{c}}} \operatorname{curl} \mathbf{E}^{0}\right|_{\Gamma^{-}} \times \mathbf{n}, \\
{\left[\mathbf{n} \times \mathbf{E}^{0}\right]_{\Gamma}=\frac{\mu_{\mathrm{m}}}{\widetilde{\kappa}_{\mathrm{m}}^{2}} \frac{\kappa_{\mathrm{c}}^{2}}{\mu_{\mathrm{c}}} \mathbf{n} \times \nabla_{\Gamma}\left(\left.\mathbf{E}^{0}\right|_{\Gamma^{-}} \cdot \mathbf{n}\right) .}
\end{gathered}
$$

The transmission condition (6e) is not classical and not easy to implement. Hence, in this paper we rewrite this condition in a new fashion easier to analyze and to implement. Transmission condition (6e) can be rewritten in terms of the tangent components of the magnetic field. Actually, since $\mathbf{J}$ vanishes in a neighborhood of the membrane, one has on $\Gamma$ the following condition:

$$
\left.\left(\operatorname{curl} \operatorname{curl} \mathbf{E}^{0}\right)\right|_{\Gamma^{-}} \cdot \mathbf{n}-\left.\kappa_{\mathrm{c}}^{2} \mathbf{E}^{0}\right|_{\Gamma^{-}} \cdot \mathbf{n}=0,
$$

from which we infer

$$
\frac{\kappa_{\mathrm{c}}^{2}}{\mu_{\mathrm{c}}} \nabla_{\Gamma}\left(\left.\mathbf{E}^{0}\right|_{\Gamma^{-}} \cdot \mathbf{n}\right)=\mathbf{n} \times \operatorname{curl}_{\Gamma} \operatorname{curl}_{\Gamma}\left(\left.\frac{1}{\mu_{\mathrm{c}}} \operatorname{curl}^{\mathbf{E}^{0}}\right|_{\Gamma^{-}}\right)_{\mathrm{T}} .
$$

Therefore, transmission condition (6e) can be rewritten into

$$
\left[\mathbf{n} \times \mathbf{E}^{0}\right]_{\Gamma}=-\frac{\mu_{\mathrm{m}}}{\widetilde{\kappa}_{\mathrm{m}}^{2}} \operatorname{curl}_{\Gamma} \operatorname{curl}_{\Gamma}\left(\left.\frac{1}{\mu_{\mathrm{c}}} \operatorname{curl} \mathbf{E}^{0}\right|_{\Gamma^{-}}\right)_{\mathrm{T}} .
$$

Note that unlike the mid-frequency case, the zeroth order term satisfies a non standard problem which links the jump of the electric field $\mathbf{E}^{0}$ to the tangential gradient of its normal component. Existence and uniqueness for such a problem is non trivial and will be discussed in the section 4.2 .

Remark 3.1 (Link with the quasistatic potential). Note that equation (6) is the extension to the electric field of the steady-state potential approximation as given by Perrussel and Poignard [14].

Actually, the quasi-static approximation consists in assuming that the solution to (6) derives from a potential, i.e. that $\mathbf{E}^{0}=-\nabla U$. Then we deduce the following partial differential equation for $U$ :

$$
\begin{aligned}
& \kappa_{\mathrm{e}}^{2} \Delta U=i \omega \mu_{\mathrm{e}} \nabla \cdot \mathbf{J}, \quad \text { in } \mathcal{O}_{\mathrm{e}}, \\
& \kappa_{\mathrm{c}}^{2} \Delta U=0, \quad \text { in } \mathcal{O}_{\mathrm{c}}, \\
& i \kappa_{\mathrm{e}} \partial_{\mathbf{n}} U=0, \quad \text { on } \partial \mathcal{O} . \\
& 7
\end{aligned}
$$


The continuity of $\mu^{-1} \kappa^{2} \mathbf{E}^{0} \cdot \mathbf{n}$ across $\Gamma$ and transmission condition (6e) lead to the following transmission conditions

$$
\begin{aligned}
& \left.\kappa_{\mathrm{e}}^{2} \partial_{\mathbf{n}} U\right|_{\Gamma^{+}}=\left.\kappa_{\mathrm{c}}^{2} \partial_{\mathbf{n}} U\right|_{\Gamma^{-}}, \\
& \frac{\widetilde{\kappa}_{\mathrm{m}}^{2}}{\mu_{\mathrm{m}}}[U]_{\Gamma}=\left.\frac{\kappa_{\mathrm{c}}^{2}}{\mu_{\mathrm{c}}} \partial_{\mathbf{n}} U\right|_{\Gamma^{-}},
\end{aligned}
$$

which is exactly the first-order approximate condition for the quasistatic potential as given by [14]. Note that the above problem is simpler than problem (6) since it has a straightforward variational formulation as shown in [14].

3.3. Influence of the position of the fictitious boundary and of weighted average of the traces on the expansion. In the above sections 3.1-3.2, we have chosen to write the condition on the boundary $\Gamma$ of the inner domain $\mathcal{O}_{c}$ but this is an arbitrary convention. Sometimes it might be interesting to place the fictitious surface on which the transmission conditions hold between the boundary of the inner domain and the surface $\Gamma^{\varepsilon}$. Actually, for any $\beta \in[0,1]$ we can define the family of surfaces that are parallel to $\Gamma$ by

$$
\Gamma_{\beta}=\left\{\mathbf{x}_{\mathbf{T}}+\beta \varepsilon \mathbf{n}\left(\mathbf{x}_{\mathbf{T}}\right), \quad \mathbf{x}_{\mathrm{T}} \in \Gamma\right\} .
$$

In addition, in the definition of $\mathbf{S}$ and $\mathbf{T}$, the surface $\Gamma^{-}$is involved but here again it is a convention, and a weighted average between $\Gamma^{+}$and $\Gamma^{-}$could have been chosen.

In order to study numerically the influence of such conventions on the convergence rate, for any $\alpha \in[0,1]$, and for any vector field $\mathbf{v}$ defined in a neighborhood of $\Gamma$, let $\left\langle\left.\mathbf{v}\right|_{\Gamma}\right\rangle_{\alpha}$ be defined by

$$
\left\langle\left.\mathbf{v}\right|_{\Gamma}\right\rangle_{\alpha}=\left.\alpha \mathbf{v}\right|_{\Gamma^{+}}+\left.(1-\alpha) \mathbf{v}\right|_{\Gamma^{-}}
$$

In this way, we obtain new transmission conditions from the transmission conditions $(5 c)-(5 d)$ in the mid-frequency case (resp. (7) in the low-frequency case) parameterized by $\alpha$ and $\beta$.

We now define the operators $\mathbf{T}_{\alpha, \beta}$ and $\mathbf{S}_{\alpha, \beta}$ as

$$
\begin{aligned}
\mathbf{T}_{\alpha, \beta}(\mathbf{E}) & =A_{\beta} \mathbf{n} \times \operatorname{curl}_{\Gamma_{\beta}} \operatorname{curl}_{\Gamma_{\beta}}\left\langle\left.\left(\frac{1}{\mu} \operatorname{curl} \mathbf{E}\right)_{\mathbf{T}}\right|_{\Gamma_{\beta}}\right\rangle_{\alpha} \\
& +B_{\beta}\left\langle\left.\left(\frac{1}{\mu} \operatorname{curl} \mathbf{E} \times \mathbf{n}\right)\right|_{\Gamma_{\beta}}\right\rangle_{\alpha}, \\
\mathbf{S}_{\alpha, \beta}(\mathbf{E}) & =C_{\beta} \overrightarrow{\operatorname{curl}_{\Gamma_{\beta}}} \operatorname{curl}_{\Gamma_{\beta}}\left\langle\left.\mathbf{E}_{\mathbf{T}}\right|_{\Gamma_{\beta}}\right\rangle_{\alpha} \\
& -D_{\beta}\left\langle\left.\mathbf{E}_{\mathbf{T}}\right|_{\Gamma_{\beta}}\right\rangle_{\alpha},
\end{aligned}
$$

where

$$
\begin{aligned}
A_{\beta} & =\frac{\mu_{\mathrm{m}}}{\kappa_{\mathrm{m}}^{2}}-\beta \frac{\mu_{\mathrm{c}}}{\kappa_{\mathrm{c}}^{2}}-(1-\beta) \frac{\mu_{\mathrm{e}}}{\kappa_{\mathrm{e}}^{2}}, \\
B_{\beta} & =\mu_{\mathrm{m}}-\beta \mu_{\mathrm{c}}-(1-\beta) \mu_{\mathrm{e}}, \\
C_{\beta} & =\frac{1}{\mu_{\mathrm{m}}}-\frac{\beta}{\mu_{\mathrm{c}}}-\frac{1-\beta}{\mu_{\mathrm{e}}}, \\
D_{\beta} & =\frac{\kappa_{\mathrm{m}}^{2}}{\mu_{\mathrm{m}}}-\beta \frac{\kappa_{\mathrm{c}}^{2}}{\mu_{\mathrm{c}}}-(1-\beta) \frac{\kappa_{\mathrm{e}}^{2}}{\mu_{\mathrm{e}}} .
\end{aligned}
$$


With such notations, for any $(\alpha, \beta) \in[0,1]^{2}$, approximate transmission conditions $(5 \mathrm{c})-(5 \mathrm{~d})$ of the mid-frequency case have to be replaced on $\Gamma_{\beta}$ by

$$
\begin{aligned}
& \mathbf{n} \times\left.\mathbf{E}^{1}\right|_{\Gamma_{\beta}^{+}} \times \mathbf{n}=\mathbf{n} \times\left.\mathbf{E}^{1}\right|_{\Gamma_{\beta}^{-}} \times \mathbf{n}+\mathbf{T}_{\alpha, \beta}\left(\mathbf{E}^{0}\right), \\
& \left.\frac{1}{\mu_{\mathrm{e}}}\left(\operatorname{curl} \mathbf{E}^{1} \times \mathbf{n}\right)\right|_{\Gamma_{\beta}^{+}}=\left.\frac{1}{\mu_{\mathrm{c}}}\left(\operatorname{curl} \mathbf{E}^{1} \times \mathbf{n}\right)\right|_{\Gamma_{\beta}^{-}}+\mathbf{S}_{\alpha, \beta}\left(\mathbf{E}^{0}\right),
\end{aligned}
$$

while transmission condition (7) of the low frequency case has to be replaced on $\Gamma_{\beta}$ by

$$
\left[\mathbf{n} \times \mathbf{E}^{0}\right]_{\Gamma_{\beta}}=-\frac{\mu_{\mathrm{m}}}{\widetilde{\kappa}_{\mathrm{m}}^{2}} \operatorname{curl}_{\Gamma_{\beta}} \operatorname{curl}_{\Gamma_{\beta}}\left(\left\langle\left.\frac{1}{\mu} \operatorname{curl} \mathbf{E}^{0}\right|_{\Gamma_{\beta}}\right\rangle_{\alpha}\right)_{\mathrm{T}} .
$$

\section{Generalized Impedance Transmission Conditions}

As seen in section 3.1, the computation of the approximate field requires to solve two similar problems which are independent of $\varepsilon$ : one for $\mathbf{E}^{0}$ and one for $\mathbf{E}^{1}$. The advantage of such approach lies in the parametric study of the problem: if one is interested in several values of the parameter $\varepsilon$, one just has to compute $\mathbf{E}^{0}$ and $\mathbf{E}^{1}$ once, and then it remains to recover the final approximation $\mathbf{E}^{\varepsilon}$ for the desired values of $\varepsilon$ with the simple operation:

$$
\mathbf{E}^{\varepsilon} \approx \mathbf{E}^{0}+\varepsilon \mathbf{E}^{1} .
$$

However if the membrane thickness is well-known, it could be interesting to solve only one problem. For such approach, the idea is to write the problem satisfied by $\mathbf{E}_{1}^{\varepsilon}=\mathbf{E}^{0}+\varepsilon \mathbf{E}^{1}$ :

$$
\begin{aligned}
& \operatorname{curlcurl} \mathbf{E}_{1}^{\varepsilon}-\kappa_{\mathrm{e}}^{2} \mathbf{E}_{1}^{\varepsilon}=i \omega \mu_{\mathrm{e}} \mathbf{J}, \text { in } \mathcal{O}_{\mathrm{e}}^{\beta}, \\
& \text { curlcurl } \mathbf{E}_{1}^{\varepsilon}-\kappa_{\mathrm{c}}^{2} \mathbf{E}_{1}^{\varepsilon}=0, \text { in } \mathcal{O}_{\mathrm{c}}, \\
& \operatorname{curl} \mathbf{E}_{1}^{\varepsilon} \times \mathbf{n}-i \kappa_{\mathrm{e}} \mathbf{n} \times \mathbf{E}_{1}^{\varepsilon} \times \mathbf{n}=0 \quad \text { on } \partial \mathcal{O},
\end{aligned}
$$

with the following transmission conditions on $\Gamma_{\beta}$

$$
\begin{aligned}
& \mathbf{n} \times\left.\mathbf{E}_{1}^{\varepsilon}\right|_{\Gamma_{\beta}^{+}} \times \mathbf{n}=\mathbf{n} \times\left.\mathbf{E}_{1}^{\varepsilon}\right|_{\Gamma_{\beta}^{-}} \times \mathbf{n}+\varepsilon \mathbf{T}_{\alpha, \beta}\left(\mathbf{E}^{0}\right), \\
& \left.\frac{1}{\mu_{\mathrm{e}}}\left(\operatorname{curl} \mathbf{E}_{1}^{\varepsilon} \times \mathbf{n}\right)\right|_{\Gamma_{\beta}^{+}}=\left.\frac{1}{\mu_{\mathrm{c}}}\left(\operatorname{curl} \mathbf{E}_{1}^{\varepsilon} \times \mathbf{n}\right)\right|_{\Gamma_{\beta}^{-}}+\varepsilon \mathbf{S}_{\alpha, \beta}\left(\mathbf{E}^{0}\right) .
\end{aligned}
$$

Here, we set $\mathcal{O}_{\mathrm{e}}^{\beta}=\left\{\mathbf{x} \in \mathcal{O}_{\mathrm{e}} \mid \operatorname{dist}(\mathbf{x}, \Gamma)>2 \beta \varepsilon\right\}$. Remarking that $\varepsilon \mathbf{S}_{\alpha, \beta}\left(\mathbf{E}^{0}\right)$ and $\varepsilon \mathbf{S}_{\alpha, \beta}\left(\mathbf{E}_{1}^{\varepsilon}\right)$ differ from a term in $\varepsilon^{2}$ (and similarly for $\varepsilon \mathbf{T}_{\alpha, \beta}\left(\mathbf{E}_{1}^{\varepsilon}\right)$ ), the final field $\mathbf{E}_{[1]}^{\varepsilon}$, which approximates $\mathbf{E}^{\varepsilon}$ at the order 2, is obtained by solving only one problem:

$$
\begin{aligned}
& \operatorname{curl} \operatorname{curl} \mathbf{E}_{[1]}^{\varepsilon}-\kappa_{\mathrm{e}}^{2} \mathbf{E}_{[1]}^{\varepsilon}=i \omega \mu_{\mathrm{e}} \mathbf{J}, \text { in } \mathcal{O}_{\mathrm{e}}^{\beta}, \\
& \operatorname{curl} \operatorname{curl} \mathbf{E}_{[1]}^{\varepsilon}-\kappa_{\mathrm{c}}^{2} \mathbf{E}_{[1]}^{\varepsilon}=0, \text { in } \mathcal{O}_{\mathrm{c}}, \\
& \operatorname{curl} \mathbf{E}_{[1]}^{\varepsilon} \times \mathbf{n}-i \kappa_{\mathrm{e}} \mathbf{n} \times \mathbf{E}_{[1]}^{\varepsilon} \times \mathbf{n}=0 \text { on } \partial \mathcal{O},
\end{aligned}
$$

with the following transmission conditions on $\Gamma_{\beta}$, called generalized impedance transmission conditions (GITC) of order 2:

$$
\begin{aligned}
& \mathbf{n} \times\left.\mathbf{E}_{[1]}^{\varepsilon}\right|_{\Gamma_{\beta}^{+}} \times \mathbf{n}=\mathbf{n} \times\left.\mathbf{E}_{[1]}^{\varepsilon}\right|_{\Gamma_{\beta}^{-}} \times \mathbf{n}+\varepsilon \mathbf{T}_{\alpha, \beta}\left(\mathbf{E}_{[1]}^{\varepsilon}\right), \\
& \left.\frac{1}{\mu_{\mathrm{e}}}\left(\operatorname{curl} \mathbf{E}_{[1]}^{\varepsilon} \times \mathbf{n}\right)\right|_{\Gamma_{\beta}^{+}}=\left.\frac{1}{\mu_{\mathrm{c}}}\left(\operatorname{curl} \mathbf{E}_{[1]}^{\varepsilon} \times \mathbf{n}\right)\right|_{\Gamma_{\beta}^{-}}+\varepsilon \mathbf{S}_{\alpha, \beta}\left(\mathbf{E}_{[1]}^{\varepsilon}\right) .
\end{aligned}
$$


Even though the theoretical derivation of the GITC models is not new, the numerical studies of these different models have not been done yet, and it is one of the novelty of this article.

4.1. Equivalent augmented formulation. In order to solve equations (11) and (6) in the same formal framework, we provide an equivalent augmented formulation. We introduce the additional unknown $\lambda$ defined as

$$
\lambda=\left\langle\frac{1}{\mu}\left(\operatorname{curl} \mathbf{E}_{[1]}^{\varepsilon} \mid \Gamma_{\beta}\right)_{\mathbf{T}}\right\rangle_{\alpha}
$$

Then the GITC for the mid-frequency case write:

$$
\begin{aligned}
\quad\left[\mathbf{n} \times \mathbf{E}_{[1]}^{\varepsilon}\right]_{\Gamma_{\beta}} & =\varepsilon\left(-A_{\beta} \operatorname{curl}_{\Gamma_{\beta}} \operatorname{curl}_{\Gamma_{\beta}} \lambda+B_{\beta} \lambda\right) \\
{\left[\frac{\mathbf{n}}{\mu} \times \operatorname{curl} \mathbf{E}_{[1]}^{\varepsilon}\right]_{\Gamma_{\beta}} } & =\varepsilon\left(-C_{\beta} \operatorname{curl}_{\Gamma_{\beta}} \operatorname{curl}_{\Gamma_{\beta}}\left\langle\left(\mathbf{E}_{[1]}^{\varepsilon}\right)_{\boldsymbol{T}}\right\rangle_{1-\alpha}+D_{\beta}\left\langle\left(\mathbf{E}_{[1]}^{\varepsilon}\right)_{\top}\right\rangle_{1-\alpha}\right),
\end{aligned}
$$

where constants $A_{\beta}, B_{\beta}, C_{\beta}, D_{\beta}$ are defined in (9).

Delourme et al. have derived in [4, Eqs. (1), (5)] a model for periodic oscillating thin layer that can be made explicit in our simpler case of homogeneous thin layer. After calculations, it falls that the model of Delourme et al. corresponds to our GITC of order 2 in the specific symmetric case $(\alpha, \beta)=(1 / 2,1 / 2)$.

Let $\mathcal{G}$ be the operator defined from $\mathrm{TH}^{-\frac{1}{2}}\left(\operatorname{div}_{\Gamma_{\beta}}, \Gamma_{\beta}\right)$ onto $\mathrm{TH}\left(\operatorname{curl}_{\Gamma_{\beta}}, \Gamma_{\beta}\right)$ by

$$
\begin{array}{ll}
\text { for any } g \in \mathrm{TH}^{-\frac{1}{2}}\left(\operatorname{div}_{\Gamma_{\beta}}, \Gamma_{\beta}\right), \quad \mathcal{G}(g)=\lambda, \quad \text { where } \lambda \text { satisfies } \\
& A_{\beta} \operatorname{curl}_{\Gamma_{\beta}} \operatorname{curl}_{\Gamma_{\beta}} \lambda-B_{\beta} \lambda=g \quad \text { on } \Gamma_{\beta},
\end{array}
$$

Note that under the assumption $\Im\left(A_{\beta}\right) \neq 0, \mathcal{G}$ is well defined and invertible. Therefore the result of Delourme et al. can be applied: in the framework of section 4.3.1, existence and uniqueness of the solution $\mathbf{E}_{[1]}^{\varepsilon} \in \mathbf{V}_{\alpha, \beta}$ to (11) hold. The proof of uniform estimates for $\mathbf{E}_{[1]}^{\varepsilon} \in \mathbf{V}_{\alpha, \beta}$ is non trivial since there is a lack of control of the divergence of the fields in the variational space $\mathbf{V}_{\alpha, \beta}$, which prevents from obtaining a compact embedding of this space in $\mathbf{L}^{2}$ (see also [5]). To overcome this difficulty (and to conclude to the well-posedness of the problem together with uniform estimates), one possibility consists of exhibiting a Helmholtz decomposition of the space $\mathbf{V}_{\alpha, \beta}$. We are confident that it is possible to adapt the proof of [5, Prop. 9] to derive a Helmholtz decomposition of $\mathbf{V}_{\alpha, \beta}$. In our configuration, this decomposition should hold without any spectral assumption (such as [5, Hyp. 6]) since $\Im\left(A_{\beta}\right) \neq 0$.

Chun et al. have derived in [2] transmission conditions when the two boundaries $\Gamma, \Gamma^{\varepsilon}$ are not reduced to a single boundary. In this approach, the membrane is not meshed, and transmission conditions are set between $\Gamma$ and $\Gamma^{\varepsilon}$. When curvature terms are removed, the second/third order transmission conditions obtained are similar to the GITC

$$
\begin{aligned}
\mathbf{n} \times\left.\mathbf{E}_{[1]}^{\varepsilon}\right|_{\Gamma^{\varepsilon}}-\mathbf{n} \times\left.\mathbf{E}_{[1]}^{\varepsilon}\right|_{\Gamma} & =\varepsilon\left(-A \operatorname{curl}_{\Gamma} \operatorname{curl}_{\Gamma} \lambda+B \lambda\right) \\
\frac{\mathbf{n}}{\mu_{\mathrm{e}}} \times\left.\operatorname{curl} \mathbf{E}_{[1]}^{\varepsilon}\right|_{\Gamma^{\varepsilon}}-\frac{\mathbf{n}}{\mu_{\mathrm{c}}} \times\left.\operatorname{curl} \mathbf{E}_{[1]}^{\varepsilon}\right|_{\Gamma} & =\varepsilon\left(-C \overrightarrow{\operatorname{curl}}_{\Gamma} \operatorname{curl}_{\Gamma}\left\langle\left(\mathbf{E}_{[1]}^{\varepsilon}\right)_{\mathbf{T}}\right\rangle_{1-\alpha}\right. \\
& \left.+D\left\langle\left(\mathbf{E}_{[1]}^{\varepsilon}\right)_{\mathbf{T}}\right\rangle_{1-\alpha}\right)
\end{aligned}
$$


with the additional unknown $\lambda$ defined as

$$
\lambda=\left\langle\frac{1}{\mu}\left(\left.\operatorname{curl} \mathbf{E}_{[1]}^{\varepsilon}\right|_{\Gamma}\right)_{\mathbf{T}}\right\rangle_{\alpha},
$$

where the constants are given by

$$
A=\frac{\mu_{\mathrm{m}}}{\kappa_{\mathrm{m}}^{2}}, \quad B=\mu_{\mathrm{m}}, \quad C=\frac{1}{\mu_{\mathrm{m}}}, \quad D=\frac{\kappa_{\mathrm{m}}^{2}}{\mu_{\mathrm{m}}} .
$$

Fourth/fifth order transmission conditions are also derived in Chun et al., involving higher-order operator. We have chosen to not consider these higher-order transmission in order to keep a comparison with only second/third order conditions.

4.2. Link with the low-frequency case. The above GITC is expected to provide an approximation of $\mathbf{E}^{\varepsilon}$ of order $O\left(\varepsilon^{2}\right)$ under the framework of mid-frequency. However, observe that if we now replace $\kappa_{\mathrm{m}}^{2}$ by $\varepsilon \widetilde{\kappa}_{\mathrm{m}}^{2}$, meaning that if we look at the low-frequency case, and if we drop the terms of order $O(\varepsilon)$ we observe that the term $\varepsilon \mathbf{S}_{\alpha, \beta}\left(\mathbf{E}_{[1]}^{\varepsilon}\right)$ has to be dropped off, while $\varepsilon \mathbf{T}_{\alpha, \beta}\left(\mathbf{E}_{[1]}^{\varepsilon}\right)$ should be identified with

$$
-\frac{\mu_{\mathrm{m}}}{\widetilde{\kappa}_{\mathrm{m}}^{2}} \operatorname{curl}_{\Gamma_{\beta}} \operatorname{curl}_{\Gamma_{\beta}}\left(\left\langle\left.\frac{1}{\mu} \operatorname{curl} \mathbf{E}_{[0]}\right|_{\Gamma_{\beta}}\right\rangle_{\alpha}\right)_{\mathrm{T}} \times \mathbf{n},
$$

where $\mathbf{E}_{[0]}$ satisfies the same problem as $\mathbf{E}^{0}$, given by (6), thanks to (7).

Therefore, from low to mid frequency, the GITC (11) provides an approximation of $\mathbf{E}^{\varepsilon}$. The order of approximation should be $O(\varepsilon)$ at low frequency (i.e. for $\left.\left|\kappa_{\mathrm{m}} / \kappa_{\mathrm{e}}\right|^{2}=O(\varepsilon)\right)$ and $O\left(\varepsilon^{2}\right)$ at mid frequency, where $\left|\kappa_{\mathrm{m}} / \kappa_{\mathrm{e}}\right| \sim 1$.

Note that in this case, (6) also falls into the above framework by changing $A_{\beta}, B_{\beta}, C_{\beta}$ and $D_{\beta}$ into

$$
A_{\beta}^{R}=\frac{1}{\varepsilon} \frac{\mu_{\mathrm{m}}}{\widetilde{\kappa}_{\mathrm{m}}^{2}}, \quad B_{\beta}^{R}=C_{\beta}^{R}=D_{\beta}^{R}=0 .
$$

However since $B_{\beta}=0$, the operator $\mathcal{G}$ defined by (12) is no longer invertible from $\mathrm{TH}^{-1 / 2}\left(\operatorname{div}_{\Gamma}, \Gamma\right)$ onto $\mathrm{TH}\left(\operatorname{curl}_{\Gamma}, \Gamma\right)$. Note however that it is invertible from the space

$$
\mathrm{TH}^{-1 / 2}\left(\operatorname{div}_{\Gamma}, \Gamma, 0\right)=\left\{\mathbf{v} \in \mathrm{TH}^{-1 / 2}\left(\operatorname{div}_{\Gamma}, \Gamma\right) \mid \operatorname{div}_{\Gamma} \mathbf{v}=0\right\},
$$

onto the space

$$
\operatorname{TH}\left(\operatorname{curl}_{\Gamma}, \Gamma, 0\right)=\left\{\mathbf{v} \in \mathrm{TH}\left(\operatorname{curl}_{\Gamma}, \Gamma\right) \mid \operatorname{div}_{\Gamma} \mathbf{v}=0\right\}
$$

and thus ad hoc modifications of the results of Delourme et al. would lead to similar existence and uniqueness results in the framework of section 4.3.2.

4.3. Generic variational formulations. Write now the variational fomulations for the two cases.

4.3.1. The mid-frequency case. We introduce a common variational framework for the GITC model (11). The functional spaces associated with $\mathbf{E}_{[1]}^{\varepsilon}$ and $\lambda$ are $\mathbf{V}_{\alpha, \beta}$ and $\mathrm{W}_{\beta}=\operatorname{TH}\left(\operatorname{curl}_{\Gamma_{\beta}}, \Gamma_{\beta}\right)$ respectively, defined as

$$
\begin{aligned}
\mathbf{V}_{\alpha, \beta}= & \left\{\mathbf{E} \in \mathbf{L}^{2}\left(\mathcal{O}_{\mathrm{c}} \cup \mathcal{O}_{\mathrm{e}}^{\beta}\right), \operatorname{curl} \mathbf{E}_{\mathrm{c}} \in \mathbf{L}^{2}\left(\mathcal{O}_{\mathrm{c}}\right), \operatorname{curl} \mathbf{E}_{\mathrm{e}} \in \mathbf{L}^{2}\left(\mathcal{O}_{\mathrm{e}}^{\beta}\right),\right. \\
& \left.\left\langle\left.\mathbf{E}_{\mathrm{T}}\right|_{\Gamma_{\beta}}\right\rangle_{1-\alpha} \in \mathrm{TH}\left(\operatorname{curl}_{\Gamma_{\beta}}, \Gamma_{\beta}\right), \mathbf{E} \times \mathbf{n} \in \mathbf{L}^{2}(\partial \mathcal{O})\right\}, \\
\mathbf{W}_{\beta}= & \operatorname{TH}\left(\operatorname{curl}_{\left.\Gamma_{\beta}, \Gamma_{\beta}\right) .}\right.
\end{aligned}
$$


Note that the functional spaces $\mathbf{V}_{\alpha, \beta}$ and $\mathrm{W}_{\beta}$ depend on $\varepsilon$ since the surface $\Gamma_{\beta}$ depends on $\varepsilon$. Then, the variational formulation for the GITC (11) writes :

Find $\left(\mathbf{E}_{[1]}^{\varepsilon}, \lambda\right) \in \mathbf{V}_{\alpha, \beta} \times \mathbf{W}_{\beta}$ such that for all $(\mathbf{U}, \xi) \in \mathbf{V}_{\alpha, \beta} \times \mathbf{W}_{\beta}$

$$
\begin{aligned}
& \int_{\mathcal{O}_{\mathrm{c}} \cup \mathcal{O}_{\mathrm{e}}^{\beta}} \frac{1}{\mu} \operatorname{curl} \mathbf{E}_{[1]}^{\varepsilon} \cdot \operatorname{curl} \overline{\mathbf{U}} \mathrm{d} \mathbf{x}-\int_{\mathcal{O}_{\mathrm{c}} \cup \mathcal{O}_{\mathrm{e}}^{\beta}} \frac{\kappa^{2}}{\mu} \mathbf{E}_{[1]}^{\varepsilon} \cdot \overline{\mathbf{U}} \mathrm{d} \mathbf{x} \\
& -i \kappa_{\mathrm{e}} \int_{\partial O} \mathbf{E}_{[1]}^{\varepsilon} \times \mathbf{n} \cdot \overline{\mathbf{U}} \times \mathbf{n} \mathrm{d} s-\int_{\Gamma_{\beta}} \mathbf{n} \times \lambda \cdot\left[\overline{\mathbf{U}_{\mathrm{T}}}\right] \mathrm{d} s \\
& +\varepsilon \int_{\Gamma_{\beta}} C_{\beta} \operatorname{curl}_{\Gamma_{\beta}}\left\langle\left(\mathbf{E}_{[1]}^{\varepsilon}\right)_{\mathrm{T}}\right\rangle_{1-\alpha} \operatorname{curl}_{\Gamma_{\beta}}\left\langle\overline{\mathbf{U}_{\mathrm{T}}}\right\rangle_{1-\alpha} \mathrm{d} s \\
& -\varepsilon \int_{\Gamma_{\beta}} D_{\beta}\left\langle\left(\mathbf{E}_{[1]}^{\varepsilon}\right)_{\mathbf{T}}\right\rangle_{1-\alpha} \cdot\left\langle\overline{\mathbf{U}_{\mathbf{T}}}\right\rangle_{1-\alpha} \mathrm{d} s \\
& =i \omega \int_{\mathcal{O}_{\mathrm{e}}} \mathbf{J} \cdot \overline{\mathbf{U}_{\mathrm{e}}} \mathrm{d} \mathbf{x}
\end{aligned}
$$

and

$$
\int_{\Gamma_{\beta}}\left[\mathbf{n} \times \mathbf{E}_{[1]}^{\varepsilon}\right] \cdot \bar{\xi} \mathrm{d} s+\varepsilon \int_{\Gamma_{\beta}} A_{\beta} \operatorname{curl}_{\Gamma_{\beta}} \lambda \operatorname{curl}_{\Gamma_{\beta}} \bar{\xi} \mathrm{d} s-\varepsilon \int_{\Gamma_{\beta}} B_{\beta} \lambda \cdot \bar{\xi} \mathrm{d} s=0 .
$$

4.3.2. Variational formulations for the low-frequency case. For the low-frequency case we define the functional spaces $\mathbf{V}_{\alpha, \beta, 0}$ and $\mathbf{W}_{\beta, 0}$ similarly to $\mathbf{V}_{\alpha, \beta}$ and $\mathbf{W}_{\beta}$ by

$$
\begin{aligned}
\mathbf{V}_{\alpha, \beta, 0}= & \left\{\mathbf{E} \in \mathbf{L}^{2}\left(\mathcal{O}_{\mathrm{c}} \cup \mathcal{O}_{\mathrm{e}}^{\beta}\right), \operatorname{curl} \mathbf{E}_{\mathrm{c}} \in \mathbf{L}^{2}\left(\mathcal{O}_{\mathrm{c}}\right), \operatorname{curl} \mathbf{E}_{\mathrm{e}} \in \mathbf{L}^{2}\left(\mathcal{O}_{\mathrm{e}}^{\beta}\right),\right. \\
& \left.\left\langle\left.\mathbf{E}_{\mathrm{T}}\right|_{\Gamma_{\beta}}\right\rangle_{1-\alpha} \in \mathrm{TH}\left(\operatorname{curl}_{\Gamma_{\beta}}, \Gamma_{\beta}, 0\right), \quad \mathbf{E} \times \mathbf{n} \in \mathbf{L}^{2}(\partial \mathcal{O})\right\} \\
\mathbf{W}_{\beta, 0}= & \operatorname{TH}\left(\operatorname{curl}_{\Gamma_{\beta}}, \Gamma_{\beta}, 0\right) .
\end{aligned}
$$

The augmented variational formulation for model (6) writes :

Find $\left(\mathbf{E}_{[0]}, \lambda\right) \in \mathbf{V}_{\alpha, \beta, 0} \times \mathbf{W}_{\beta, 0}$ such that for any test-vector field $\mathbf{U} \in \mathbf{V}_{\alpha, \beta, 0}$ :

$$
\begin{aligned}
& \int_{\mathcal{O}_{\mathrm{c}} \cup \mathcal{O}_{\mathrm{e}}^{\beta}} \frac{1}{\mu} \operatorname{curl} \mathbf{E}_{[0]} \cdot \operatorname{curl} \overline{\mathbf{U}} \mathrm{d} \mathbf{x}-\int_{\mathcal{O}_{\mathrm{c}} \cup \mathcal{O}_{\mathrm{e}}^{\beta}} \frac{\kappa^{2}}{\mu} \mathbf{E}_{[0]} \cdot \overline{\mathbf{U}} \mathrm{d} \mathbf{x}-i \kappa_{\mathrm{e}} \int_{\partial O} \mathbf{E}_{[0]} \times \mathbf{n} \cdot \overline{\mathbf{U}} \times \mathbf{n} d s \\
& -\int_{\Gamma_{\beta}} \mathbf{n} \times \lambda \cdot\left[\overline{\mathbf{U}_{\mathrm{T}}}\right] \mathrm{d} s=i \omega \int_{\mathcal{O}_{\mathrm{e}}} \mathbf{J} \cdot \overline{\mathbf{U}_{\mathrm{e}}} \mathrm{d} \mathbf{x},
\end{aligned}
$$

where $\lambda$ satisfies the variational formulation for any smooth enough test-function $\xi \in \mathrm{W}_{\beta, 0}$ :

$$
-\int_{\Gamma_{\beta}}\left[\mathbf{n} \times \mathbf{E}_{[0]}\right] \cdot \bar{\xi} \mathrm{d} s-\frac{\mu_{m}}{\widetilde{\kappa}_{\mathrm{m}}^{2}} \int_{\Gamma_{\beta}} \operatorname{curl}_{\Gamma_{\beta}} \lambda \operatorname{curl}_{\Gamma_{\beta}} \bar{\xi} \mathrm{d} s=0 .
$$

4.3.3. Uniqueness. One easily has the following uniqueness result for the above variational formulations.

Lemma 4.1 (Uniqueness result). Assume that $\sigma$ is strictly positive in $\mathcal{O}$ and suppose that a solution $(\mathbf{E}, \lambda)$ to the variational formulation (19) (resp. to (17)) exists. Then it is necessarily unique. 
Proof. We prove the results for (19) when $\beta=0$, the proof of (17) is exactly the same, mutatis mutandis.

If a solution $(\mathbf{E}, \lambda)$ exists with $\mathbf{J}$ equal to zero, then necessarily, by taking as test-couple $(\mathbf{U}, \xi)=(\mathbf{E}, \lambda)$, and using the conjugate form of (19b), one infers

$$
\begin{aligned}
& \int_{\mathcal{O}_{\mathrm{c}} \cup \mathcal{O}_{\mathrm{e}}} \frac{1}{\mu}\left|\operatorname{curl} \mathbf{E}_{[0]}\right|^{2} d x-\omega^{2} \int_{\mathcal{O}_{\mathrm{c}} \cup \mathcal{O}_{\mathrm{e}}}\left(\epsilon+i \frac{\sigma}{\omega}\right)\left|\mathbf{E}_{[0]}\right|^{2} d x-i \kappa_{\mathrm{e}} \int_{\partial O}\left|\mathbf{E}_{[0]} \times \mathbf{n}\right|^{2} d s \\
& -\frac{\mu_{m}}{\left|\widetilde{\kappa}_{\mathrm{m}}^{2}\right|^{2}} \widetilde{\kappa}_{\mathrm{m}}^{2} \int_{\Gamma}\left|\operatorname{curl}_{\Gamma} \lambda\right|^{2} d s=0
\end{aligned}
$$

Taking the imaginary part of the above equality, one infers that $\mathbf{E}_{[0]}$ and $\operatorname{curl}_{\Gamma} \lambda$ vanish. Then, since

$$
\operatorname{curl}_{\Gamma} \lambda=0 \quad \text { and } \quad \operatorname{div}_{\Gamma} \lambda=0,
$$

and due to the assumption on $\Gamma$, we infer that $\lambda$ identically vanishes, hence the uniqueness.

\section{NumERiCAL Simulations}

5.1. Numerical method and models. The variational formulation (17) is discretized with highorder hexahedral edge elements of the first kind (see [3] for more details). Here, a fifth-order approximation and curved elements will be used throughout all the experiments. Edge elements have been chosen since they are adapted to the variational formulation (17), and they are more natural for the resolution of Maxwell's equations. They don't need a specific regularization as for nodal finite elements, or penalty terms as for Discontinuous Galerkin methods. As illustrated in [5], using hexahedral elements is more efficient than tetrahedral elements when the geometry can be easily meshed with hexahedral elements (as in Figure 2). Elements of the first kind are used because hexahedral elements of the second kind generate spurious modes (see [3]). Finally, the objective of the paper is to compare different models for thin layers, the discretization method used to solve time-harmonic Maxwell's equations is not of major interest.

When using edge elements, the following surface finite element matrix $\left(\varphi_{i}\right.$ being a function test associated with the boundary $\Gamma_{\beta}$ )

$$
M_{i, j}=\int_{\Gamma_{\beta}} \mathbf{n} \times \varphi_{j} \cdot \bar{\varphi}_{i}
$$

is singular, so that the global finite element matrix will be non-invertible as soon as $B_{\beta}$ of (9) is equal to 0 . In order to ensure the invertibility of the global matrix, we add to $B_{\beta}$ a penalty term :

$$
B_{\beta} \leftarrow B_{\beta}+\varepsilon \delta
$$

$\delta$ is chosen equal to $10^{-7}$ in all the numerical experiments. This value has been chosen in order to avoid too small pivots when the direct solver tries to factorize the finite element matrix. By choosing such a value, the finite element matrix is always correctly factorized, and the numerical solution is close enough to the solution with $\delta=0$. The numerical results presented can be considered unchanged if $\delta$ would have been chosen smaller.

We think that by choosing more carefully the finite element space for $\lambda$, for instance a divergencefree finite element space (such that $\operatorname{div} \lambda=0$ is ensured by construction) like the space $\operatorname{TH}\left(\operatorname{curl}_{\Gamma}, \Gamma, 0\right)$, the surface finite element matrix $M$ may become invertible, and the penalty term could be dropped. Another attractive solution would be to use nodal finite elements for $\lambda$, this solution has not been investigated.

The following models will be compared : 


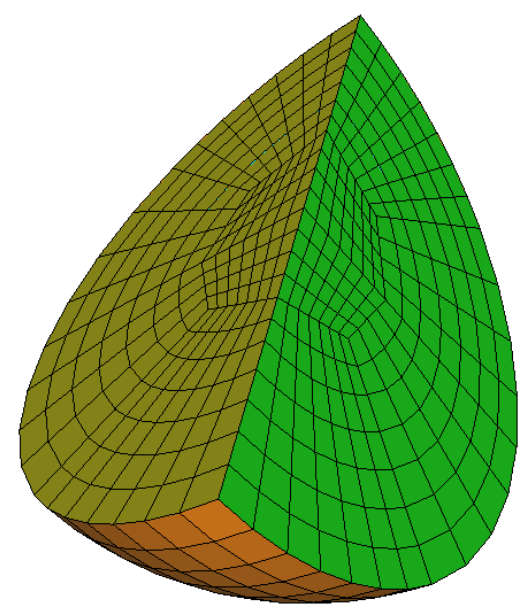

FIGURE 2. Example of hexahedral mesh used for the biological cell

- Symmetric model (variational formulation (17) with $\beta=0.5$ ) : this is the model obtained by Delourme et al. [4]. In this model, the membrane is replaced by the boundary $\Gamma_{1 / 2}$, which is the median boundary between $\Gamma$ and $\Gamma^{\varepsilon}$.

- GITC model (variational formulation (17) with $\beta=0$ ) : this model is similar to the previous one, but the membrane is replaced by the boundary $\Gamma$ instead of $\Gamma_{1 / 2}$.

- Resistive model (variational formulation (19) with $\beta=0$ ) : this model is similar to a GITC model where coefficients $A_{\beta}, B_{\beta}, C_{\beta}, D_{\beta}$ are simplified, assuming that the frequency is small.

- $\mathbf{E}^{0}+\varepsilon \mathbf{E}^{1}$ model (solving problems (2) and (5)) : this is the model proposed in [7], where $\mathbf{E}^{0}$ is computed, then $\mathbf{E}^{1}$ is computed with a right-hand-side depending on $\mathbf{E}^{0}$. Once $\mathbf{E}^{0}$ and $\mathbf{E}^{1}$ computed, the approximated solution is given as $\mathbf{E}^{0}+\varepsilon \mathbf{E}^{1}$

- Chun model (using conditions (13) with $\alpha=0.5$ ) : this is the model obtained by Chun et al. [2]. In this model, the membrane is not meshed, and the two boundaries $\Gamma, \Gamma^{\varepsilon}$ are distinct. The variational formulation is similar to (17) where the coefficients $A, B, C, D$ are given by (14).

Note that, as it can be seen, all the models are giving similar transmissions conditions (only expression of coefficients $A_{\beta}, B_{\beta}, C_{\beta}, D_{\beta}$ differ with respect to each position of the membrane): all these models can be seen as different way to derive the asymptotic expansion of the electromagnetic field solution to (1).

The "exact" solution will be the numerical solution $u_{\text {exact }}^{h}$ computed on a mesh where the membrane is present. $u_{\text {exact }}^{h}$ solves the problem (1), where $h$ is the mesh size. We denote $u^{h}$ the solution of an approximate model (e.g. the solution of symmetric model is obtained by considering formulation (17)). $u_{\text {exact }}^{0}$ and $u^{0}$ are the limits of $u_{\text {exact }}^{h}$ and $u^{h}$ when $h$ tends to 0 . The relative $L^{2}$ error that will be displayed in the sequel is given as:

$$
\text { Relative } L^{2} \text { error }=\frac{\left\|u_{\text {exact }}^{h}-u^{h}\right\|_{2}}{\left\|u_{\text {exact }}^{h}\right\|_{2}}
$$


The triangular inequality provides:

$$
\left\|u_{\text {exact }}^{h}-u^{h}\right\|_{2} \leq\left\|u_{\text {exact }}^{h}-u_{\text {exact }}^{0}\right\|_{2}+\left\|u^{h}-u^{0}\right\|_{2}+\left\|u_{\text {exact }}^{0}-u^{0}\right\|_{2}
$$

We have observed that the numerical error $\left\|u_{\text {exact }}^{h}-u_{\text {exact }}^{0}\right\|_{2}$ obtained for the exact solution is approximately the same as the numerical error $\left\|u^{h}-u^{0}\right\|_{2}$ obtained when computing each model. Therefore, when the numerical error will be mentioned without detail, it refers as well for the computation of the exact solution as for the computation of any model. The term $\left\|u_{\text {exact }}^{0}-u^{0}\right\|_{2}$ is called the model error.

5.2. Validation. The models are validated for the scattering of a sphere with the following parameters

$$
\begin{aligned}
& \epsilon_{m}=3.5, \quad \mu_{\mathrm{m}}=2.0, \quad \sigma_{m}=0.05 \\
& \epsilon_{c}=2.0, \quad \mu_{\mathrm{c}}=1.5, \quad \sigma_{c}=0.02 \\
& \epsilon_{\mathrm{e}}=1.0, \quad \mu_{\mathrm{e}}=1.0, \quad \sigma_{e}=0.0
\end{aligned}
$$

The main objective of this validation is to test the different models on a simple case satisfying

$$
\epsilon_{m} \neq \epsilon_{c} \neq \epsilon_{\mathrm{e}}, \quad \mu_{\mathrm{m}} \neq \mu_{\mathrm{c}} \neq \mu_{\mathrm{e}}, \quad \sigma_{m} \neq \sigma_{c} \neq \sigma_{e}
$$

An additional objective is to see if choosing $\alpha=0$ or $\alpha=1$ is better than $\alpha=0.5$. The source is imposed via the Silver-Müller condition $(\mathbf{J}=0)$ :

$$
\operatorname{curl} \mathbf{E} \times \mathbf{n}-i \kappa_{\mathrm{e}} \mathbf{n} \times \mathbf{E} \times \mathbf{n}=\operatorname{curl} \mathbf{E}^{\mathrm{inc}} \times \mathbf{n}-i \kappa_{\mathrm{e}} \mathbf{n} \times \mathbf{E}^{\mathrm{inc}} \times \mathbf{n}
$$

where the incident plane wave is equal to

$$
\mathbf{E}^{\text {inc }}=e^{i \kappa_{\mathrm{e}} x} \mathbf{e}_{z}
$$

The wave vector is oriented along $\mathbf{e}_{z}$, the polarization along $\mathbf{e}_{x}$. The real part of the solution is displayed in Fig. 3 for $\varepsilon=0.04$. As it can be observed in Fig. 4, the choice $\alpha=0.5$ gives more accurate results than the other tested values of $\alpha$ for this model case.

The GITC model and symmetric model both provide a convergence in $O\left(\varepsilon^{2}\right)$, the symmetric model being more accurate than GITC for this case. Chun's model provides a convergence in $O\left(\varepsilon^{3}\right)$, and is the most accurate. Expected rates of convergence are numerically observed on this simple case, therefore these models are correctly implemented. We think that the symmetric model is more accurate than GITC model, because when

$$
\epsilon_{e}=\epsilon_{c}, \quad \mu_{e}=\mu_{c}, \quad \sigma_{e}=\sigma_{c},
$$

the symmetric model provides a convergence in $O\left(\varepsilon^{3}\right)$. Since chosen values are not that far from satisfying those equalities, the symmetric model is more accurate. In a second experiment, we change values of $\mu$ such that the coefficient $B_{\beta}$ is null for $\beta=\frac{1}{2}$ :

$$
\mu_{\mathrm{m}}=1.5, \quad \mu_{\mathrm{c}}=2.0, \quad \mu_{\mathrm{e}}=1 .
$$

For this experiment, the relative $L^{2}$ error is computed versus the penalty parameter $\delta$ for $\varepsilon=0.01$, the result is displayed in Figure 5.

In this figure, the relative "Delta" error is also displayed, this error is given as

$$
\text { Relative Delta error }=\frac{\left\|u^{h, \delta}-u^{h, 0}\right\|_{2}}{\left\|u^{h, 0}\right\|_{2}}
$$



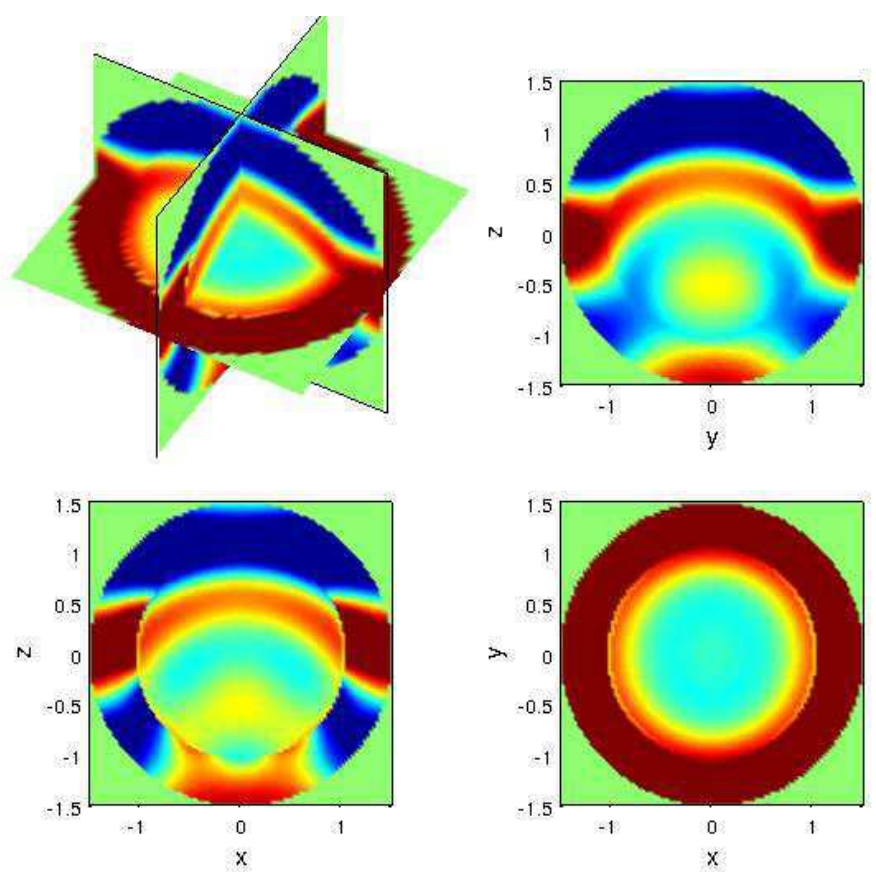

FIGURE 3. Real part of the total field for the sphere (exact solution). $\varepsilon=0.04$

where $u^{h, 0}$ is the numerical solution for $\delta$ equal to $10^{-10}$. It can be observed that the value $\delta=10^{-7}$ is well inside the region where $\delta$ does not influence the relative $L^{2}$ error. For this example, $\delta$ can be chosen up to 0.5 without influencing too much the relative $L^{2}$ error.

5.3. Electric parameters of biological cell. In this section, $\alpha$ will be taken equal to 0.5 since this value gave the best results in the validation case. We study here the case of a biological cell, assumed to be a sphere whose radius is equal to $10 \mu \mathrm{m}$ with the following parameters

$$
\begin{aligned}
& \epsilon_{m}=10.0, \quad \mu_{\mathrm{m}}=1.0, \quad \sigma_{m}=5.0 e-7 \\
& \epsilon_{c}=80.0, \quad \mu_{\mathrm{c}}=1.0, \quad \sigma_{c}=1.0 \\
& \epsilon_{\mathrm{e}}=80.0, \quad \mu_{\mathrm{e}}=1.0, \quad \sigma_{e}=0.5
\end{aligned}
$$

These parameters are close to the real parameters measured in biological cells. $\mu$ is identically equal to 1 , as a result $B_{\beta}$ and $C_{\beta}$ are equal to 0 for all models except Chun's model. The conductivity of the membrane $\sigma_{m}$ is small compared to $\sigma_{e}, \sigma_{c}$. This fact also induces two regimes: a low-frequency regime where

$$
\omega^{2} \epsilon_{m}+i \sigma_{m} \omega \text { much smaller than } \omega^{2} \epsilon_{e}+i \sigma_{e} \omega_{e}
$$

and a high-frequency regime these quantities are approximatively of the same order. The source is here a Gaussian distribution:

$$
\mathbf{J}=\left\{\begin{array}{l}
\beta_{J} \exp \left(-\alpha_{J} r^{2}\right) \mathbf{e}_{x}, \text { if } r<r_{J} \\
0 \text { otherwise }
\end{array}\right.
$$




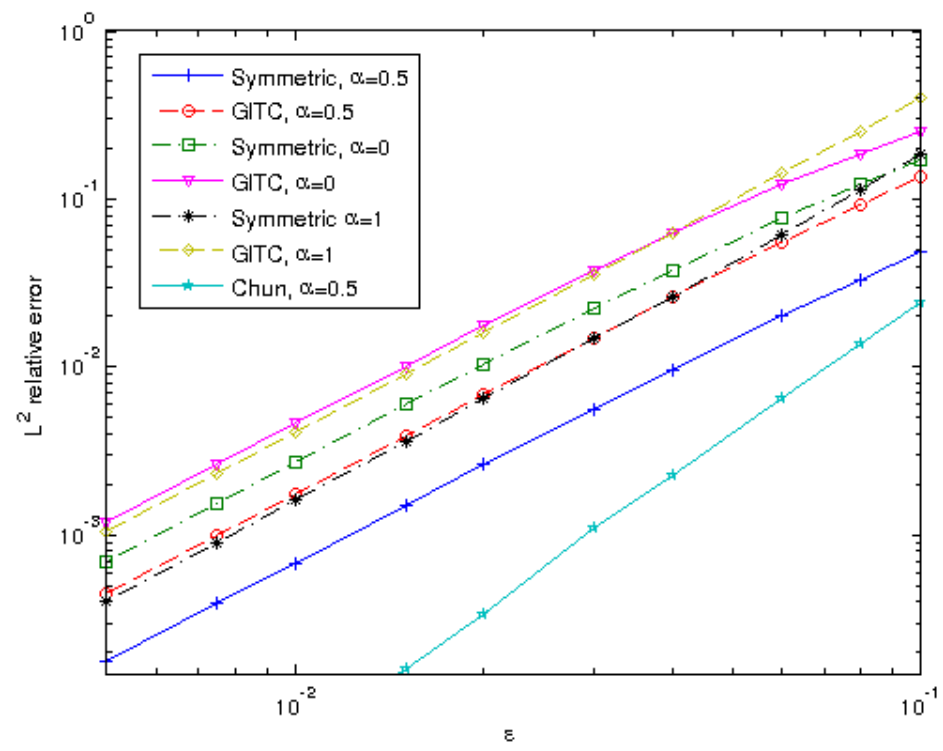

FIGURE 4. Relative $L^{2}$ error versus thickness $\varepsilon$ for the symmetric model and GITC for different values of $\alpha$

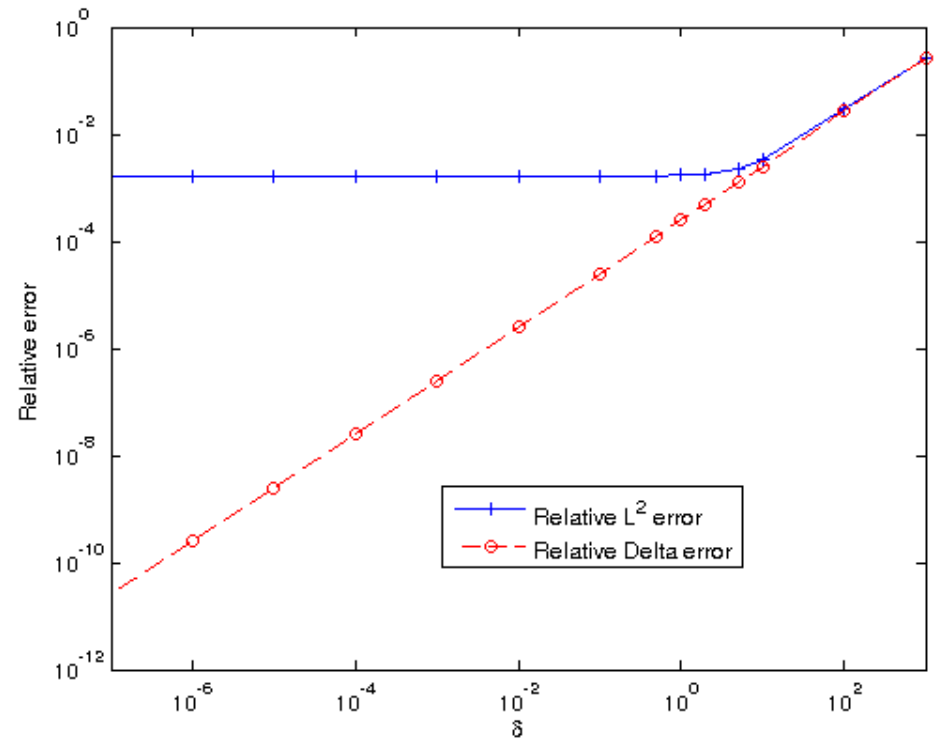

FIGURE 5. Relative $L^{2}$ error and Delta error versus penalty parameter $\delta$ for $\varepsilon=0.01$.

$\alpha_{J}$ and $\beta_{J}$ are two constants, $r_{J}$ is equal to $4 \mu \mathrm{m}$. The outer boundary is a sphere of radius 20 $\mu \mathrm{m}$, and the center of the gaussian is located at $(0,0,15)$. The radius of the membrane is taken equal to $10 \mu \mathrm{m}$, the thickness of the membrane is taken equal to $0.01 \mu \mathrm{m}$. 
To illustrate the difference between the two regimes (low-frequency and high-frequency), The exact solution is displayed for two frequencies : $10 \mathrm{Mhz}, 100 \mathrm{Mhz}$ in Figure 6. In this figure, the solution is displayed along the axis Oz. For a frequency equal to $100 \mathrm{Mhz}$, the solution is almost continuous, whereas for a frequency equal to $10 \mathrm{Mhz}$, the solution presents an important discontinuity through the membrane. This illustration shows that there are two asymptotics with respect to $O(\varepsilon)$, an asymptotic in high-frequency regime, which is the continuous solution (the term $\mathbf{E}^{0}$ is dominant), and an asymptotic in low-frequency regime, which is computed by the resistive model. This last asymptotic is indeed discontinuous throughout the interface $\Gamma$.

The symmetric model provides a solution that fits perfectly to the exact solution (see Figure 6). The solution computed by the symmetric model is not represented for $100 \mathrm{Mhz}$ to keep a figure clear enough, but the obtained solution fits to the exact solution for this frequency also. The 3-D solution is displayed for the low frequency case (10 Mhz) in Figure 7.

In Figure 8, the different models are compared versus the frequency and the following regimes are observed:

- High-frequency regime (frequency above $10^{12} \mathrm{~Hz}$ ) : the $L^{2}$ error increases with the frequency because the wavelength decreases whereas the membrane thickness is constant. The error obtained with the $\mathbf{E}^{0}+\varepsilon \mathbf{E}^{1}$ model and the resistive model are close, the resistive model being less accurate. The error obtained with the GITC model, symmetric and Chun's model is similar, the two last models being more accurate. The symmetric and

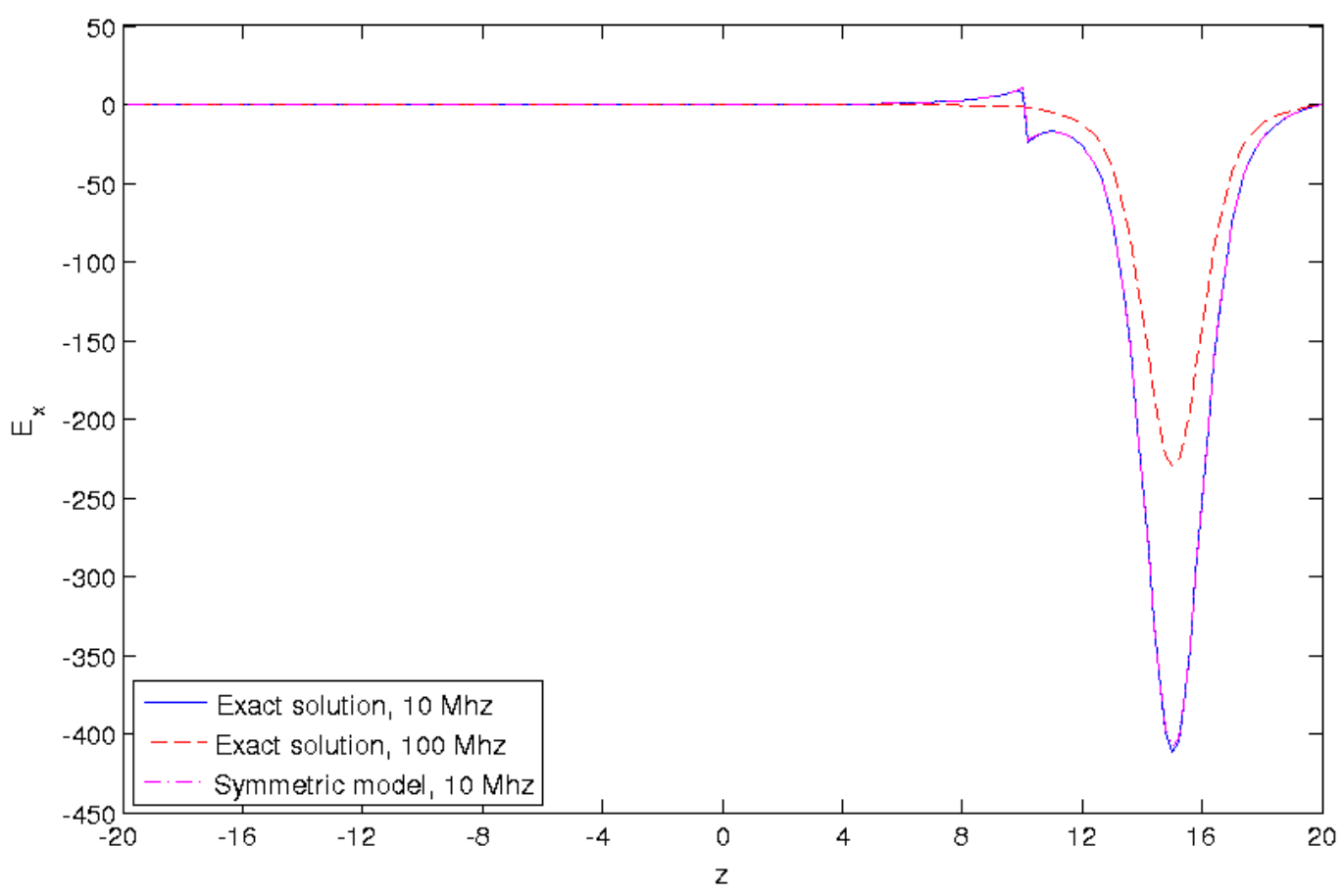

FIGURE 6. Exact solution for the biological cell for a frequency equal to 10 and $100 \mathrm{Mhz}$, and solution obtained with the symmetric model. Real part of $E_{x}$. 
Chun's model give the same error, because the error is here dominated by the numerical error (due to the discretization) and not by the model error.

- Mid-frequency regime (frequency between $10^{8} \mathrm{~Hz}$ and $10^{12} \mathrm{~Hz}$ ) : the $L^{2}$ error of all the models, except the $\mathbf{E}^{0}+\varepsilon \mathbf{E}^{1}$ model, is dominated by the numerical error. As a result, all the models are giving the same relative $L^{2}$ error, approximately $2 \cdot 10^{-4}$. This level can be decreased if the mesh is refined. In this regime, the $\mathbf{E}^{0}+\varepsilon \mathbf{E}^{1}$ model gives an error which increases with the frequency, the error goes beyond $100 \%$. This behaviour can be seen as a locking phenomenon for the $\mathbf{E}^{0}+\varepsilon \mathbf{E}^{1}$ model, since the constant of convergence is worsening when the frequency is decreasing, whereas the other models seem robust for small frequencies. In this regime, there is no particular advantage to use a model over another one (except the $\mathbf{E}^{0}+\varepsilon \mathbf{E}^{1}$ model of course).

- Low-frequency regime (frequency below $10^{8} \mathrm{~Hz}$ ) : the $L^{2}$ error of all the models increases when the frequency decreases. This phenomenon stems from the fact that the numerical method is not robust for small frequencies. The finite element matrix also tends to a singular matrix. The numerical method is rather appropriate for mid-frequency cases than for low-frequency cases. It can be observed that Chun's model is more sensitive to this problem than other models.

In Figure 9, the models are compared versus the thickness for a high-frequency case $(f=$ $5 \cdot 10^{12} \mathrm{~Hz}$. For this case, the symmetric model, Chun's model, $\mathbf{E}^{0}+\varepsilon \mathbf{E}^{1}$ and GITC seem to produce a convergence in $O\left(\varepsilon^{2}\right)$, whereas the resistive model converges in $O(\varepsilon)$. The model $\mathbf{E}^{0}+\varepsilon \mathbf{E}^{1}$ is also clearly less accurate than GITC.

For low-frequency cases, all the models show a first-order convergence (see Figure 10), but because of the locking phenomenon in the $\mathbf{E}^{0}+\varepsilon \mathbf{E}^{1}$ model, this model seems to not converge. A convergence should be observed for a very small thickness. The error obtained with Chun's model is sometimes unexpectedly high, we think that this is due to the sensitivity of Chun's model to the lack of robustness of the numerical method for small frequencies.

\section{CONCLUSiON}

In this paper we have studied numerically the electromagnetic field in a domain with thin layer, which presents two different behaviors: at low frequency, when the ohmic currents are dominant, the domain is highly contrasted with a very resistive thin membrane, while at mid frequencies, for which the currents of displacement are prominent the material is softly contrasted. This is the main feature of biological cells.

To avoid the meshing of the thin membrane, five approximations of the electromagnetic field have been used, we have detailed the drawbacks and advantages of each model:

- Symmetric model (variational formulation (17) with $\beta=0.5$ ). This model is accurate for all frequencies. However, the position of the median interface $\Gamma_{1 / 2}$ (see Sec. 3.3) must be known, the position of this interface depends on $\varepsilon$, therefore a different mesh is needed for each value of $\varepsilon$.

- GITC model (variational formulation (17) with $\beta=0$ ). This model is accurate for all frequencies, but less accurate than symmetric model or Chun's model. If the boundary of the cytoplasm $\Gamma$ is known, the transmission conditions are directly posed on this interface. There is no need to construct the boundary $\Gamma^{\varepsilon}$, therefore the same mesh can be used for different values of $\varepsilon$. 

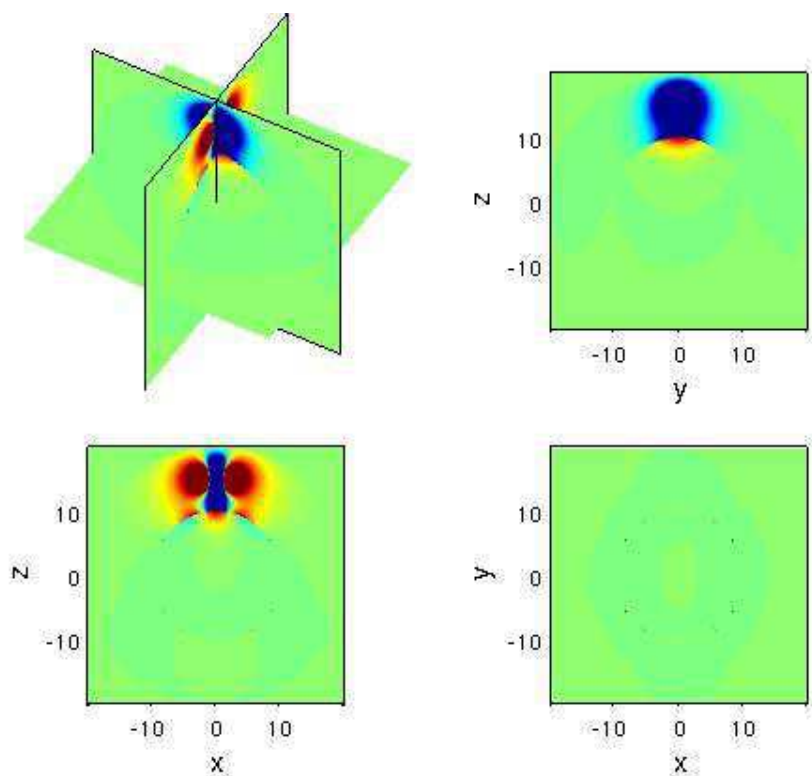

FIGURE 7. Exact solution for $10 \mathrm{Mhz}$. Real part of $E_{x}$.

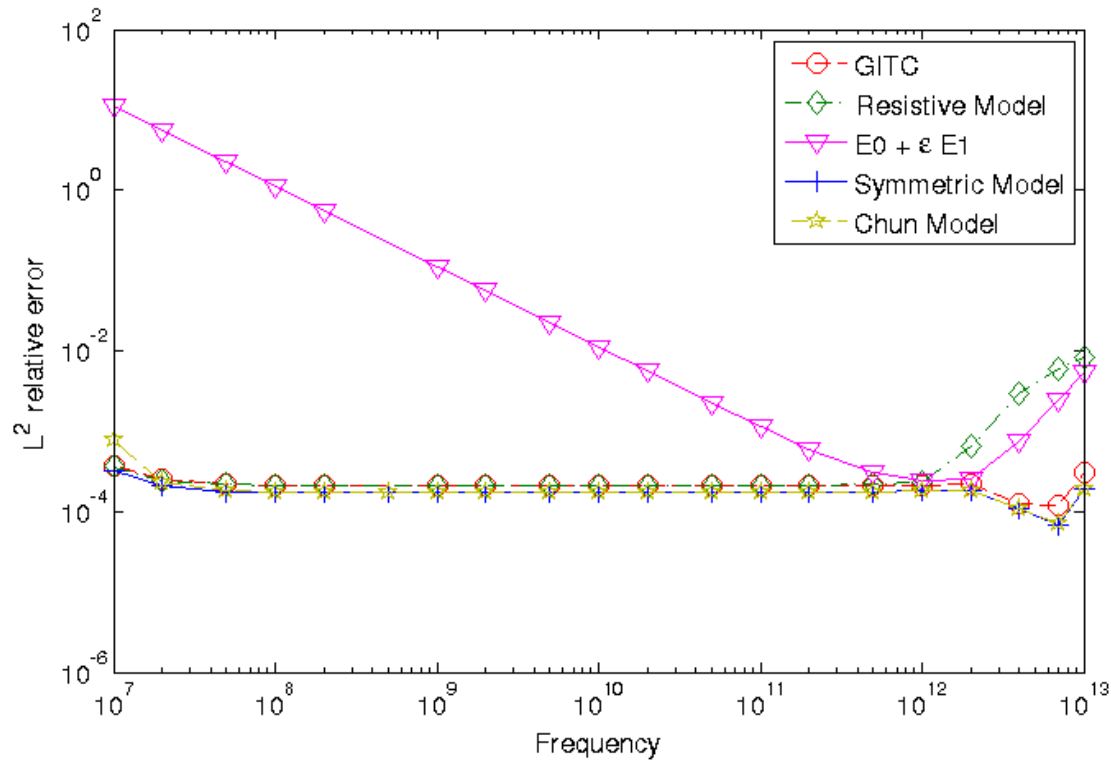

FIGURE 8. Relative $L^{2}$ error of the different models versus the frequency (in $\mathrm{Hz}$ ).

- Resistive model (variational formulation (19) with $\beta=0$ ). This model is accurate for the low-frequency case, but is inaccurate for high frequency. This model is the most simple to consider since $B_{\beta}=C_{\beta}=D_{\beta}=0$. 


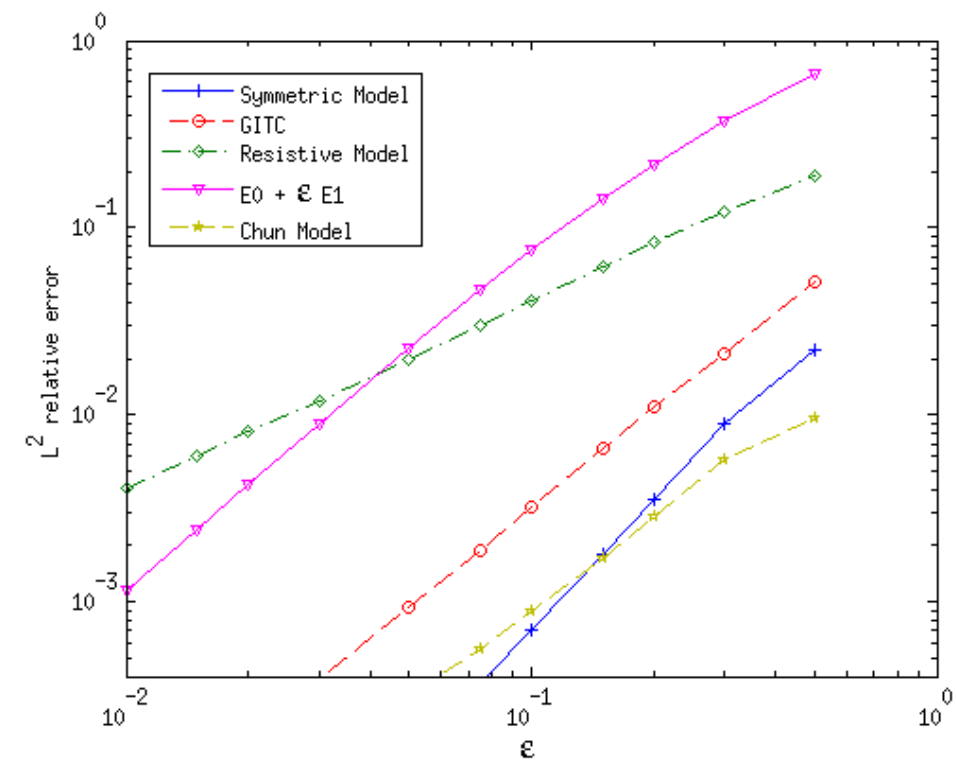

FIGURE 9. Relative $L^{2}$ error of the different models versus the thickness (in $\mu m$ ). Frequency equal to $5 \mathrm{THz}$.

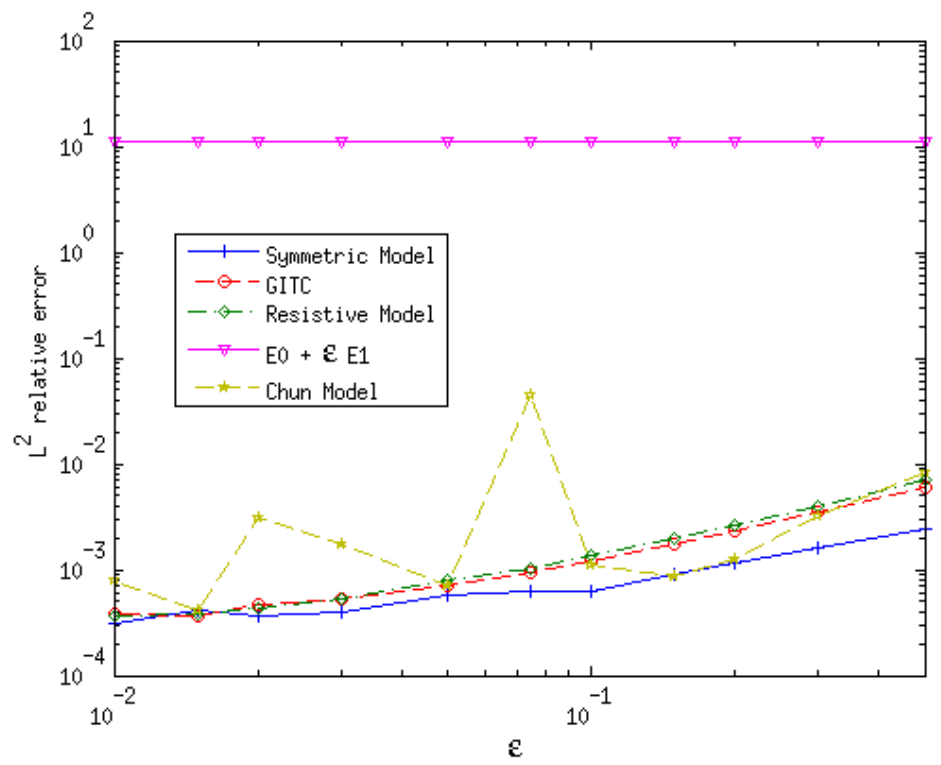

FIGURE 10. Relative $L^{2}$ error of the different models versus the thickness (in $\mu \mathrm{m})$. Frequency equal to $10 \mathrm{MHz}$.

- $\mathbf{E}^{0}+\varepsilon \mathbf{E}^{1}$ model (solving problems (2) and (5)). This model provides a poor accuracy, with a locking phenomenon for the low-frequency case. In our opinion, this model should be avoided. However, it may be interesting if one wants to study the influence of the 
membrane thickness on the electric field by changing the value of $\varepsilon$ for mid-frequencies. Actually, the coefficients $\mathbf{E}^{0}$ and $\mathbf{E}^{1}$ do not depend on $\varepsilon$ and they have to be computed only once: the change of $\varepsilon$ appearing in the sum $\mathbf{E}_{1}^{\varepsilon}=\mathbf{E}^{0}+\varepsilon \mathbf{E}^{1}$, whereas for other models, a change in the value of $\varepsilon$ makes it necessary to compute the electric field again.

- Chun model (using conditions (13) with $\alpha=0.5$ ). This model is accurate for all frequencies. A first drawback is the necessity to mesh two distinct boundaries $\Gamma$ and $\Gamma^{\varepsilon}$. This last boundary depends on $\varepsilon$, therefore a different mesh is needed for each value of $\varepsilon$. Another drawback over other models is that the finite element matrix is non-symmetric (except for planar interfaces) due to the two separated boundaries. The coefficients $A, B, C, D$ are always positive contrary to other models for which the positivity depends on the coefficients $\epsilon, \mu$ in the cytoplasm and the exterior. As a result, Chun's model is stable in the time-domain (as proved in [2]) whereas other models are generally not stable. In [4], a proposed solution to ensure positive coefficients for the symmetric model consists of separating the interface into two distinct boundaries with a distance equal to $\alpha \varepsilon$, and the coefficients are consequently modified and become positive for $\alpha$ large enough, giving stable transmission conditions in time-domain. In our opinion, time-domain simulations should prefer Chun's model, since it is always stable and accurate as well.

6.1. Acknowledgment. The authors thank the reviewers for their comments and advices that improved this paper.

Experiments presented in this paper were carried out using the PLAFRIM experimental testbed, being developed under the Inria PlaFRIM development action with support from LABRI and IMB and other entities: Conseil Régional d'Aquitaine, FeDER, Université de Bordeaux and CNRS (see https://plafrim.bordeaux.inria.fr/). Computer time for this study was provided by the computing facilities MCIA (Mésocentre de Calcul Intensif Aquitain) of the Université de Bordeaux and of the Université de Pau et des Pays de l'Adour.

This study has been carried out in the frame of the Investments for the future Programme IdEx Bordeaux CPU (ANR-10-IDEX-03-02). C.P is partly funded by ANR projects INTCELL (ANR 2010-BLAN-916) and MEMOVE (ANR 2011 BS01 006 01).

\section{REFERENCES}

[1] G. Caloz, M. Dauge, E. Faou, and V. Péron. On the influence of the geometry on skin effect in electromagnetism. Computer Methods in Applied Mechanics and Engineering, 200(9-12):1053-1068, 2011.

[2] S. Chun, H. Haddar, and J. S. Hesthaven. High-order accurate thin layer approximations for time-domain electromagnetics, Part II: transmission layers. J. Comput. Appl. Math., 234(8):2587-2608, 2010.

[3] C. Cohen and M. Duruflé. Non spurious spectral-like element methods for Maxwell's equations. Journal of Computational Mathematics, 25:282-304, 2007.

[4] B. Delourme, H. Haddar, and P. Joly. On the Well-Posedness, Stability And Accuracy Of An Asymptotic Model For Thin Periodic Interfaces In Electromagnetic Scattering Problems. Mathematical Models and Methods in Applied Sciences, 2013.

[5] M. Duruflé. Intgration numrique et lments finis d'ordre lev appliqus aux quations de Maxwell en rgime harmonique. PhD thesis, Universit Paris IX-Dauphine, 2006.

[6] M. Durufle, V. Péron, and C. Poignard. Thin Layer Models for Electromagnetism. In Waves 2011: The 10th International Conference on Mathematical and Numerical Aspects of Waves, pages 687-690, Vancouver, Canada, July 2011.

[7] M. Duruflé, V. Péron, and C. Poignard. Time-harmonic Maxwell equations in biological cells-the differential form formalism to treat the thin layer. Confluentes Math., 3(2):325-357, 2011. 
[8] E.C. Fear and M.A. Stuchly. Modelling assemblies of biological cells exposed to electric fields. IEEE Trans Biomed Eng, 45(10):1259-1271, Oct 1998.

[9] E.C. Fear and M.A. Stuchly. A novel equivalent circuit model for gap-connected cells. Phys Med Biol, 43(6):1439-1448, Jun 1998.

[10] K.R. Foster and H.P. Schwan. Dielectric properties of tissues and biological materials: a critical review. $C R C$ in Biomedical Engineering, 17(1):25-104, 1989.

[11] O. Kavian, M. Leguèbe, C. Poignard, and L. Weynans. "Classical” Electropermeabilization Modeling at the Cell Scale. Journal of Mathematical Biology, 2012.

[12] S. Muñoz, J.L. Sebastián, M. Sancho, and J.M. Miranda. Transmembrane voltage induced on altered erythrocyte shapes exposed to RF fields. Bioelectromagnetics, 25(1):631-633 (electronic), 2004.

[13] J.-C. Nédélec. Acoustic and electromagnetic equations, volume 144 of Applied Mathematical Sciences. SpringerVerlag, New York, 2001. Integral representations for harmonic problems.

[14] R. Perrussel and C. Poignard. Asymptotic expansion of steady-state potential in a high contrast medium with a thin resistive layer. Applied Mathematics and Computation, to appear, 2013.

[15] C. Poignard. Asymptotics for steady-state voltage potentials in a bidimensional highly contrasted medium with thin layer. Math. Methods Appl. Sci., 31(4):443-479, 2008.

[16] C. Poignard. About the transmembrane voltage potential of a biological cell in time-harmonic regime. ESAIM: Proceedings, 26:162-179, 2009.

[17] C. Poignard. Approximate transmission conditions through a weakly oscillating thin layer. Math. Meth. App. Sci., 32(4):435-453, 2009.

[18] C. Poignard, P. Dular, R. Perrussel, L. Krähenbühl, L. Nicolas, and M. Schatzman. Approximate conditions replacing thin layer. IEEE Trans. on Mag., 44(6):1154-1157, 2008.

[19] G. Pucihar, T. Kotnik, B. Valič, and D. Miklavčič. Numerical determination of transmembrane voltage induced on irregularly shaped cells. Ann Biomed Eng, 34(4):642-652, Apr 2006.

[20] J.L. Sebastián, S. Muñoz, M. Sancho, and J.M. Miranda. Analysis of the influence of the cell geometry and cell proximity effects on the electric field distribution from direct rf exposure. Phys. Med. Biol., 46:213-225 (electronic), 2001.

[21] J. Teissié, M. Golzio, and M.P. Rols. Mechanisms of cell membrane electropermeabilization: A minireview of our present (lack of?) knownledge. Biochimica et Biophysica Acta, 1724:270-280, 2005.

(M. Duruflé) Team Magique-3D, INRIA BordeauX-Sud-Ouest, Institut de Mathématiques de BordeAuX, CNRS UMR 5251 \& UniVERSITÉ DE BordeAuX1, 351 COURS DE LA LibÉRATION, 33405 TALENCE CEDEX, France.

E-mail address, M. Duruflé: marc.durufle@inria.fr

(V. Péron) Team Magique-3D, INRIA BordeauX-Sud-Ouest, LMAP CNRS UMR 5142 \& Université De PAu et des Pays de L'Adour, France.

E-mail address, V. Péron: victor.peron@inria.fr

(C. Poignard) Team MC2, INRIA BordeauX-Sud-Ouest, Institut de Mathématiques de BordeauX, CNRS UMR 5251 \& UNIVERSITÉ DE BORdEAUX1, 351 COURS DE LA LibÉRATION, 33405 TALENCE CEDEX, FRANCE.

E-mail address, C. Poignard: clair.poignardeinria.fr 